\title{
Graphene Nano-Fiber Composites for Enhanced Neuronal Differentiation of Human Mesenchymal Stem Cellst
}

Sujata Mohanty ( $\nabla$ drmohantysujata@gmail.com )

All India Institute of Medical Sciences https://orcid.org/0000-0002-0047-4914

Krishan Gopal Jain

All India Institute of Medical Sciences

Sonali Rawat

All India Institute of Medical Sciences

Deepika Gupta

Indian Institute of Technology Delhi

\section{Pawan Raghav}

All India Institute of Medical Sciences

\section{Rituparna Chaudhuri}

All India Institute of Medical Sciences

Pinky .

All India Institute of Medical Sciences

\section{Adeeba Shakeel}

All India Institute of Medical Sciences

\section{Varun Arora}

Indian Institute of Technology Delhi

Harshita Sharma

All India Institute of Medical Sciences

Debika Debnath

University of Bridgeport

Ankarao kalluri

University of Bridgeport

Ashwini Agrawal

Indian Institute of Technology Delhi

Manjeet Jassal

Indian Institute of Technology

Amit Kumar Dinda

All India Institute of Medical Sciences

Prabir Patra 


\section{Research}

Keywords: Graphene, Mesenchymal stem Cells, Neuronal differentiation, Dopaminergic neurons, Electrospinning, nanofibers.

Posted Date: December 1st, 2020

DOl: https://doi.org/10.21203/rs.3.rs-115775/v1

License: (c) (1) This work is licensed under a Creative Commons Attribution 4.0 International License. Read Full License 


\section{Abstract}

Graphene-based nanocomposites have been extensively employed to design biomimetic platforms epitomizing the structural and functional complexity of the tissue with increased robustness and physiological relevance. The adhesive and mechanical cues provided by such nanocomposite microenvironment kindles the cell fate decisions. Owing to their differentiation and regenerative potential, Human Mesenchymal Stem Cells (hMSCs) have proven to be a promising candidate for treating several neurodegenerative disorders. However, their degree of differentiation and its reproducibility is often jeopardized by multiple levels of heterogeneity, thereby compromising their translational utilization. Baffled at this crossroad, we designed a one-step approach to electrospin Poly-caprolactone (PCL) nanocomposite, with varying graphene concentrations, to capture, for the first time, the realms of their biocompatible and anisotropic characteristics, providing biomimetic platforms for improved differentiation of human bone marrow-derived MSCs (hMSCs) into neurons. Interestingly, PCL having $0.05 \%$ graphene (PCL-G0.05) showcased an ideal nano-topography with an unprecedented combination of guidance stimuli and substrates cues, aiding in enhanced differentiation of hMSCs into dopaminergic neurons (DA). These newly differentiated DA neurons were characterized at gene, protein, and functional levels and were seen to exhibit unique neuronal arborization, enhanced intracellular $\mathrm{Ca}^{2+}$ influx, and dopamine secretion, thereby opening new horizons for pre-clinical and clinical applications.

\section{Introduction}

The increasing burden of neurodegenerative diseases concomitantly escalates the morbidity, thus affecting the patient's quality of life. Since the adult brain and spinal cord have a limited regenerative capacity, most neurodegenerative diseases like Parkinson's result in the loss of functional cell population, such as dopaminergic (DA) neurons [1, 2]. The replenishment of these degenerated DA neurons via stem cell therapy has been proven to be a potential therapeutic approach to treat Parkinson's disease[3].

From already reported different types of stem cells like embryonic stem cells (ESCs), neural precursor cells (NPCs), or induced pluripotent stem cells (iPSCs), Mesenchymal Stem Cells (MSCs) have proven to be the best candidate 4 . A plethora of studies has also shown that MSCs are immunologically naïve, can be used for transplantation in an allogenic setting, and can trans-differentiate into cells of ectodermal and endodermal lineages $[5,6,7]$. However, poor homing, low engraftment and differentiation efficiency, are the various facets of malady, which fades their regenerative ability. This can be overcome by modulating, enhancing, and directing their differentiation either by using various chemical cues like drugs, growth factors, or by switching to economical and more exogenous repair technology of employing biomaterials. An array of scaffolds have been fabricated using synthetic polymers such as Polylactic acid (PLA), poly (glycolic acid) (PGA), Polyethylene terephthalate (PET) and natural polymers like silk, chitosan, and collagen that have been studied as a carrier for cells keeping in mind to imitate the extracellular matrix [8]. Among them, polycaprolactone $(\mathrm{PCL})$ has shown great potential because of its biocompatibility, slow degradation, mechanical strength, and its versatility to take any shape or form. Reports have shown that PCL can be very easily electrospun into nanofibers having interconnected 
porous structure that can support cell attachment along with neurite outgrowth [9]. However, its hydrophobic surface and poor electro-conductivity limits its use as an efficient cell carrier [10]. Being electro-active cells, neurons yearn for a platform that can respond to the electrical stimuli mimicking an ideal environment providing topographical, electrical, and chemical cues for their better adhesion, morphology, proliferation, and differentiation [11]. Since it is the surface conductivity of the scaffolds that majorly influence such cellular responses $[12,13]$, combining PCL with a material having superior electrical conductivity can tune their properties to regulate better neuronal differentiation, thereby amplifying their excitability. Owing to the unique combination of biocompatibility and highest chargecarrier motility of any known material [14], graphene (G), emerged as the best-chosen filler, for neuronal tissue engineering [15]. It is a two-dimensional allotrope of carbon with atoms arranged in a honeycomblike framework. Its mechanical strength, high surface area, and electro-conductive properties, significantly enhance the differentiation of stem cells to neuronal, cardiac, and osteogenic lineages in comparison to cells cultured on scaffolds without graphene [15-18]. Apart from providing conductive properties, graphene is also known to adsorb proteins and other biomolecules from the cell culture medium and make them available to growing cells [14, 19]. Additionally, it contains a lot of wrinkles and ripples on their surface that makes it convenient for the cells to attach [20]. Graphene in its native form is highly hydrophobic and hence many studies have used its reduced form (graphene oxide (GO) [21-24] or other carbon nanomaterials like carbon nanotubes (CNTs). However, GO has a compromised electronic conductance as compared to naïve graphene ${ }^{14}$, whereas, CNTs are proven to induce toxicity to the cells [25].

Recently, there were reports quoting the efficiency of PCL and graphene combination for nerve tissue engineering, however, they either focussed on using neural stem cells (NSCs) [26], or have only investigated the neuronal or associated population growth overlooking the functional characterization of the differentiated neurons [9].

Keeping these points in mind and in the quest to unlock the potential role of PCL and graphene in the differentiation of human Bone marrow-derived MSCs (hMSCs) into functional DA neurons, we established a one-step, facile fabrication protocol for the synthesis of electrospun PCL-Graphene (PCL-G) nanocomposites. We also performed various characterization studies of the prepared membranes. The as-synthesized nanocomposite scaffolds constituted a nano-to-micro framework, had excellent biocompatibility, large surface-to-volume ratio, suitable topography, and optimum mechanical strength that offered a robust platform for reproducible and efficient differentiation of hMSCs. Interestingly, hMSCs differentiated on PCL-G nanocomposites had superior functionality over cells cultured on PCL only and assessed by $\mathrm{Ca}^{2+}$ and dopamine secretion estimation, thereby, making them more appropriate for clinical applications.

\section{Materials And Methods}

\section{Fabrication of nanofibrous scaffolds}


PCL (Mn:70,000-90,000 g/mol, Sigma), single layer nano-platelet graphene (Cheap tube Inc.USA), and methylthiazolyldiphenyl-tetrazolium bromide (MTS) were purchased from Promega (Madison, WI, USA). Acetone and glutaraldehyde solution ( $25 \mathrm{wt} \%$ in H2O) were supplied from Merck (Darmstadt, Germany). All the chemicals were of analytical grade and used without additional treatment. PCL-G nanofiber composites were prepared by the electrospinning method using graphene dispersed PCL solution made in acetone. Nanofibrous composites with four different compositions were prepared, as shown in Table1. Briefly, graphene powder was sonicated for $90 \mathrm{~min}$ in acetone to obtain stable graphene dispersions with different concentrations of $0.1,0.5$, and $1.0 \mathrm{mg} / \mathrm{ml}$. A weighed amount of PCL pellets was dissolved in graphene dispersions under vigorous stirring to obtain $15 \%(\mathrm{w} / \mathrm{w})$ solution. The PCL solutions with or without graphene were placed into a syringe with a 22-gauge needle and electrospun onto an aluminium surface, which was positioned vertically, at a flow rate of $0.5 \mathrm{~mL} / \mathrm{h}$. A 17-kV voltage was applied with a high voltage power supply, and a $15 \mathrm{~cm}$ working distance was utilized for electrospinning. The resultant non-woven nanofibrous mats were air-dried in a fume hood for $24 \mathrm{~h}$ and then stored in a desiccator. The samples of desired dimensions were cut for various experiments.

Table 1. Composition of PCL and PCL-G nanocomposites

\begin{tabular}{|lllll|}
\hline S.No. & $\begin{array}{l}\text { Coding } \\
\text { Name }\end{array}$ & $\begin{array}{l}\text { PCL } \\
(\% w / w)\end{array}$ & $\begin{array}{l}\text { Graphene (G) concentrations } \\
(\mathbf{m g} / \mathrm{ml})\end{array}$ & $\begin{array}{l}\text { G filler (\%w/v of PCL } \\
\text { solution) }\end{array}$ \\
\hline 1 & PCL & 15 & 0.0 & 0.0 \\
\hline 2 & PCL-G0.01 & $\begin{array}{l}\sim 15 \\
(14.99)\end{array}$ & 0.1 & $0.01 \%$ \\
\hline 3 & PCL-G0.05 & $\begin{array}{l}\sim 15 \\
(14.95)\end{array}$ & 0.5 & $0.05 \%$ \\
\hline 4 & PCL-G0.1 & $\sim 15(14.9)$ & 1.0 & $0.1 \%$ \\
\hline
\end{tabular}

\section{Characterization of nanofibrous scaffolds}

The morphology and fiber dimensions of PCL and PCL-G nanocomposites were examined by scanning electron microscopy (SEM) (EVO, 18 Research, Zeiss, Germany). Before imaging, all samples were vacuum dried and coated with $1 \mathrm{~nm}$ gold. SEM images were taken under $30 \mathrm{kV}$ voltage and $8 \mathrm{~mm}$ working distance. Diameters of individual fiber in the scaffolds were measured directly from the SEM images with $10 \mathrm{k} X$ magnification using Image $\mathrm{J}$ software $(\mathrm{NIH}$, Bethesda). Fiber diameter was measured from ten randomly selected areas $(n=5)$, and the mean \pm standard deviation was used to interpret the data. SEM images of the cross-section of the scaffolds were also taken to measure scaffold thickness. High-resolution transmission electron microscopy (HRTEM) of PCL-G0.1 nanofibers was done to confirm the incorporation of graphene within the PCL nanofibers. Sample for PCL-G0.1 was prepared by directly spinning the solution on a carbon-coated copper grid and observed using TEM (FEI Tecnai TF20, Oregon, USA). The dispersion of graphene in the PCL scaffolds was evaluated using Fourier transform Infrared 
spectroscopy (FTIR, Thermo Scientific ${ }^{\text {TM }}$ Nicolet $^{\text {TM }}$ iS50 FTIR Spectrometer) and Raman spectroscopy (Renishaw, INVIA confocal micro dispersive Raman spectrometer, laser excitation at $514 \mathrm{~nm}$ ). X-ray diffraction (XRD) analysis of graphene and nanofibrous scaffolds was performed on X'Pert PRO, PANalytical, Netherlands. The water contact angle for all the samples was measured by a modified ASTM D 5946-04 static sessile drop method. In this, a water droplet of $30 \mu \mathrm{L}$ was put on the flat substrate using a micro-syringe, and high-resolution images were captured using a Canon DSLR 70D camera. The image was processed, and the average contact angle of 3 droplets was reported at 3 random locations on each PCL and PCL-G nanocomposite nanofiber mats. The surface topography and roughness of PCL and PCLG0.05 nanofiber scaffolds were investigated using Atomic force microscopy (AFM), TOSCA Tm 400 AFM (Anton Paar, Austria) in air, under tapping mode. Thermal characterization of nanofibrous scaffolds was done using thermal series Thermogravimetric analysis (TGA) Q-500 and Differential scanning calorimetry (DSC) Q-200 from TA Instruments (New Castle, DE, USA). The rheological studies were carried out via small-amplitude oscillatory shear experiments by applying fixed stress of $1.0 \mathrm{~Pa}$, on a AR 500 Rheometer (TA Instruments, Surrey, England). Cone-plate geometry $\left(2^{\circ}\right)$ was used to carry out the measurements, and silicone oil with wet sponges was utilized as a solvent trap. The frequency dependence of storage $\left(G^{\prime}\right)$ and loss modulus (G") studies were carried out at room temperature. The tensile properties of PCL and PCL-G nanocomposite scaffolds membrane were measured using an Instron 3365 Microtester (High Wycombe, UK), at a crosshead speed of $50 \mathrm{~mm} / \mathrm{min}$ and a load cell of $10 \mathrm{~N}$ capacity.

\section{Human Bone Marrow derived Mesenchymal Stem Cells (hMSCs) expansion and characterization}

Ethical clearance was taken from Institutional committee for Stem cell Research (ICSCR-/87/18(0)) before initiating the experiments. Cryopreserved hMSCs, were revived and cultured in hMSC culture medium (CM) consisting of Low Glucose Dulbecco's Modified Eagle medium (LG-DMEM, Gibco, USA) supplemented with $10 \%$ fetal bovine serum (FBS, Gibco, USA) 1\% L-glutamine and $1 \%$ penicillin/streptomycin (Gibco, USA). Cells were maintained in $25-\mathrm{cm}^{2}$ flasks at $37^{\circ} \mathrm{C}$ and placed in a humidified incubator in the presence of $5 \% \mathrm{CO} 2$, during which the medium was replaced every 2 days. Cells from $3^{\text {rd }}$ - 4 th passage were used in the present study. They were expanded and characterized as published earlier $[27,28]$.

\section{Scaffolds preparation for cell culture}

PCL and PCL-G nanocomposite mats were cut into square specimens of $6 \mathrm{~mm} \times 6 \mathrm{~mm}$ size. These scaffolds were sterilized by immersing in $70 \%$ ethanol for $30 \mathrm{~min}$, followed by three sterile phosphate buffer saline (PBS) washings of 10 min each. The sterilized specimens were placed in a 48 well tissue culture plate and soaked in $\mathrm{CM}$ overnight for preconditioning of scaffolds. The above procedure was used to prime the scaffolds for all the experiments.

\section{hMSCs Viability/cytotoxicity and attachment over nanofibrous Scaffolds}

Morphology and distribution of viable hMSCs seeded on the PCL and PCL-G nanocomposite scaffolds were examined after $24 \mathrm{~h}$ using Live/Dead staining Kit (Thermo Scientific, USA). Briefly, hMSCs were 
seeded ( $2 \times 10^{4}$ cells/scaffold) on sterile PCL and PCL-G nanocomposite scaffolds and incubated in a $\mathrm{CO}_{2}$ incubator with CM. After $24 \mathrm{~h}$, scaffolds were washed with PBS and incubated for 20 min with CalceinAM and EthD-I staining solution. After staining, the cells were washed with PBS and visualized using a Confocal laser scanning microscope (TCS SP8, Leica, Germany). The experiment was repeated thrice.

Cell proliferation assay. Cell proliferation assay was performed using FxCycle ${ }^{\mathrm{TM}} \mathrm{PI} / \mathrm{RNase}$ (Molecular probes, Thermofisher, USA) reagent as per the manufacturer's protocol. Briefly, hMSCs were seeded ( $4 \times 10^{4}$ cells/scaffold) on sterile PCL and PCL-G nanocomposite scaffolds and were incubated in a $\mathrm{CO}_{2}$ incubator with CM. Cells were trypsinized, fixed, and stained with $\mathrm{FxCycle}^{\mathrm{TM}} \mathrm{PI} / \mathrm{RNase}$ solution at day 1, 3, and 5. Stained cells were acquired by flow cytometry, and the data was analyzed using 6.0 Diva software. The experiment was repeated thrice.

\section{Measurement of Mitochondrial health in hMSCs cultured on nanofibrous scaffolds}

hMSCs were seeded at a density of 60,000 cells per well on PCL and PCL-G nanocomposites and cultured in $\mathrm{CM}$ for 5 days. Cells were trypsinized and plated on 24-well XF-24 plates (Seahorse Biosciences, Billerica, MA, USA) to estimate Oxygen consumption rate (OCR) using the XFe24 Extracellular Flux Analyzer (Seahorse Biosciences). The experiment was repeated thrice.

\section{Estimation of Extracellular matrix (ECM) secretion by hMSCs on nanofibrous scaffolds}

To understand the interaction of cells with the scaffolds, the deposition of extracellular matrix was evaluated by staining collagen protein. Mouse anti-Collagen (Abcam, USA) antibody at 1:100 was incubated in $1 \% \mathrm{BSA}$ at $4^{\circ} \mathrm{C}$ overnight. To study the morphological changes, cells were stained with F-actin (Rhodamine Phalloidin at 1:100 dilution for 40 mins at room temperature(RT), (Life Technologies, USA)). After successive washing steps in PBS, the anti-collagen treated groups were incubated with anti-mouse Alexa Fluor 488(1:500) at RT for 1h, followed by PBS wash. Nucleus was stained with DAPI (1:4000) for 3 min. After staining, the cell-scaffold constructs were washed twice with PBS and examined under a confocal laser scanning microscope (SP5, Leica, Germany).

\section{Histocompatibility assay}

The in-vivo tissue compatibility of PCL and PCL-G nanocomposites was evaluated by the deep wound model. Deep wound incision was created on the dorsal side of Wistar rats $(n=1)$ with an area of $1 \times 1 \mathrm{~cm}$ (approx.).The wound area was analyzed by histological staining. The rats were observed on day 3 , day 7 \& day 15 after the implant. On day 15, after the implant, skin samples were collected \& stored in 10\% formalin. After the dehydration with a series of ethanol, the samples were embedded in paraffin wax with a sectioned thickness of $5 \mu \mathrm{m}$. Hematoxylin \& Eosin (H\&E) and Immunohistochemistry (IHC) staining were performed to study the expression of IL-6 (CST, 1:200). 


\section{Neuronal differentiation hMSCs on nanofibrous scaffolds}

To evaluate the role of graphene in stem cell differentiation into the neuronal lineage, hMSCs were suspended in CM and then seeded onto sterilized PCL and PCL-G nanocomposite scaffolds at a density of $2 \times 10^{4}$ cells per well for $24 \mathrm{~h}$ for cell attachment. After $24 \mathrm{~h}$, cells were maintained in CM (un-induced) and in Neuronal induction medium (neurobasal medium supplemented with $0.5 \mathrm{mM}$ glutamine, $1 \%$ penicillin/streptomycin (Gibco, USA), 2\% B-27 serum-free supplement and 10nM Endothelial growth factor (EGF) (Peprotech, USA), 10nM Fibroblast growth factor (FGF-2) (Peprotech, USA) and 10nM oxysterol (Sigma Aldrich, USA) for 14 days at $37^{\circ} \mathrm{C}$ with $5 \% \mathrm{CO}_{2}$. hMSCs cultured over cover glass served as a control in both the uninduced and induced culture conditions.

\section{Characterization of differentiated neurons}

Cell morphology on the nanofibrous scaffold: Morphology of cells seeded on the PCL and PCL-G nanocomposite scaffolds was examined by SEM. Samples were collected after 14 days differentiation process and fixed with $2.5 \%$ glutaraldehyde, washed 3 times with PBS, and finally stored at $-80^{\circ} \mathrm{C}$. Samples were freeze-dried using lyophilizer. Dried samples were mounted over aluminium stubs and sputter-coated with gold prior to imaging with a Zeiss scanning electron microscope (Co-operation Zeiss, Leica, Cambridge, UK) at 5 KVA in secondary electron imaging mode.

\section{Immunofluorescence}

Cells differentiated into neurons were characterized by immunofluorescence using neuron-specific markers (TH, MAP-2, and beta-III tubulin). After 14 days of culture in both uninduced and induced conditions, cells grown over scaffolds or glass coverslips were fixed with $4 \%$ paraformaldehyde for 20 min at RT and then washed in PBS. Fixed cells were permeabilized in 0.05\% Triton-X100 (Sigma-Aldrich, St Louis, MO, USA) for 20 min, followed by PBS wash. Nonspecific antibody binding was blocked with $2 \%$ BSA (HiMedia, India) for 20 min at RT before PBS wash. To characterize the hMSCs differentiated into neuronal cells were then incubated with mouse-anti-MAP-2 (Microtubule Associated Protein) (Santa Cruz, 1:100), beta-III tubulin (Thermofisher Scientific) (1:100) and for DA neurons they were incubated with Rabbit-anti-TH protein (Tyrosine Hydroxylase) (Thermofisher Scientific) (1:100) in 1\% BSA at $4{ }^{\circ} \mathrm{C}$ overnight. After subsequent washing in PBS, the cells were incubated in anti-mouse Alexa Fluor 488 and Texas Red (1:500) at RT for $1 \mathrm{~h}$, respectively, followed by a PBS wash and DAPI nucleus staining (1:4000) for 3 min. After staining, the cell-scaffold constructs were washed twice with PBS and examined under a Confocal laser scanning microscope (SP5, Leica, Germany).

\section{Morphological characterization of neuronal differentiated cells (neurite length \& cell alignment)}

hMSCs differentiated into neurons on PCL, and PCL-G nanocomposites nanofibrous scaffolds were imaged using a confocal laser scanning microscope. For each condition, the number of analyzed neurite $(n)$ is reported. Neurite lengths were estimated with the ImageJ software (NIH, Bethesda, MD, USA). 5 fields from 3 images were taken to calculate the neurite length $(n=15)$. 


\section{Quantification of cell over fiber orientation}

Fluorescent images of hMSCs differentiated neurons stained for MAP-2 and confocal images were analyzed with Fiji (http://fiji.sc/Fuji). It calculates the number of histograms indicating the number of cells in a given direction. A flat histogram shows the comprehensive isotropic content, though a histogram with a peak confirms orientation. A Gaussian function computes the directionality parameter to measure the orientation of the cells.

\section{Measurement of intracellular calcium ions $\left(\mathrm{Ca}^{+2}\right)$ in neuronal differentiated cells over nano-fibrous scaffolds}

Change in the concentration of intracellular $\mathrm{Ca}^{+2}$ was studied by $\mathrm{Ca}^{+2}$ imaging in hMSCs induced for 14 days in all study groups, as published earlier. Briefly, hMSCs after 14days of neuronal induction were stained with $4 \mu \mathrm{M}$ of Fura-2-red AM dye. After washing thrice with Hanks' Balanced Salt Solution (HBSS), the cells were activated using $56 \mathrm{mM} \mathrm{KCl}$ solution. The time-lapse recording was made at $405 \mathrm{~nm}$ and $488 \mathrm{~nm}$ for 5 minutes. Baseline readings were obtained before adding $\mathrm{KCl}$ solution to the cells. The experiment was performed using a Leica Confocal Microscope (TCS SP8, Leica Germany). The ratio of fluorescence at both the wavelengths was obtained, and the respective graph was plotted. The experiment was repeated thrice. The data was analyzed using Leica LAS AF 520 software.

\section{Measurement of dopamine released by differentiated cells on nanofibrous scaffolds}

Cell culture supernatants $(1 \mathrm{ml})$ from both cover glass and nanofibrous scaffolds were collected at $14^{\text {th }}$ day to estimate the dopamine release in control and induced conditions. To further estimate the inducible release of dopamine, the cells were stimulated with $56 \mathrm{mM} \mathrm{KCl}$ (Sigma) for $5 \mathrm{~min}$, in 4-(2-hydroxyethy 1)-1piperazineethanesulfonic acid (HEPES)-buffered salt solution consisting of $\mathrm{NaCl} 130, \mathrm{KCl} 5.4, \mathrm{MgCl} 20.8$, $\mathrm{CaCl} 2$ 1.8, $\mathrm{Cl}$-130.6, HEPES 20 and Glucose $15(\mathrm{mM})$, at $\mathrm{pH} 7.4$ at $37^{\circ} \mathrm{C}$. The dopamine secretion level was measured by a Dopamine ELISA kit (DA ELISA, Elabscience Biotechnology Inc., USA) according to the manufacturer's protocol. Absorbance was subsequently measured at $450 \mathrm{~nm}$ recorded with Gen5 1.08.4 software using EL 800 multi-well microplate reader (BioTek, USA). The experiment was repeated thrice.

\section{Molecular dynamics (MD) simulation studies between $\mathrm{G}$ and F-actin}

Modelling and Docking: The three-dimensional (3D) structure of graphene, single layer, $5 \mathrm{~nm} \times 5 \mathrm{~nm}$ was generated using the Visual Molecular Dynamics (VMD) tool. The structural comparison of various F-actin structures revealed that $6 \mathrm{ANU}$ is the most suitable 3D structure which have complete sequence length (1375) with best resolution ( $7 \AA$ ). The native 3D structure of F-actin was download from Protein Data Bank (PDB code: $6 \mathrm{ANU}$ ) and docked with graphene by the Autodock vina. The graphene was treated as receptor while F-actin was considered as ligand and prepared for docking by adding hydrogen and Gasteiger charges, and assigned AD4 type atomic radii. The best docked complex was obtained by 
calculating electrostatic grid map using center grid box $(23.947,24.815$ and 0.000 points) with number of points in 126126126 direction.

Prediction of interaction between $\mathrm{G}$ and F-actin: MD simulation MD simulations were performed for complex monomer F-actin nanocomposite system of a single layer, $5 \mathrm{~nm}$ x $5 \mathrm{~nm}$ Graphene. After energy minimization, the solvated systems were pre-equilibrated by initially for $100 \mathrm{ps}$ at a constant pressure of 1 bar and a temperature of $300 \mathrm{~K}$ with V-rescale methods. Subsequently, the $100 \mathrm{~ns}$ production runs were carried out for system with adopted time step of $0.002 \mathrm{ps}$ and $50000000 \mathrm{n}$ steps. The final molecular dynamics simulations were conducted for nanocomposite in the constant-NPT ensemble, with the mean pressure at $1 \mathrm{~atm}$, using a Parrinello-Rahman barostat, and the mean temperature fixed at $300 \mathrm{~K}$ using a V-rescale thermostat. All simulations were performed using the gromacs-2019.1 package with periodic boundary conditions. The Optimized Protein Liquid Simulations (OPLS) force field was applied to represent the intermolecular and intramolecular interactions of each system. The TIP4P water model was used to simulate the system. The system was neutralized by adding $12 \mathrm{Na}+$ ions. Particle mesh Ewald (PME) was used to calculate the electrostatic interactions with a PME order of 4. Lennard-Jones interactions were truncated at $1 \mathrm{~nm}$. Neighbor lists were updated every 10fs using a list cut-off radius of 1 $\mathrm{nm}$. Periodic boundary conditions (PBC) was applied for all systems. Bond constraints were solved using the Linear Constraint Solver (LINCS) algorithm using the 4 lincs order. In the used graphene model, the carbon atoms at the edges were capped with hydrogen atoms to avoid the unsaturated boundary effect. All the analysis was accomplished using the tools of GROMACS.

\section{Histocompatibility assay}

The tissue biocompatibility of PCL and PCL-G nanocomposites was evaluated by creating the excision wound model wherein the excision was created on the dorsal side of Wistar rats $(n=1)$ with an area of $1 \times 1$ $\mathrm{cm}$ (approx.). Scaffolds were then implanted over these wounds. The area of implant in the wound was biopsied at day 15 to observe the tissue reaction. All the biopsies were fixed in $10 \%$ buffered formalin processed in automated tissue processor (Histokinette, Lieca biosystems, Germany), embedded in paraffin. Five micron thick serial paraffin sections were cut from representative areas of the wound. The sections were stained with Hematoxylin \& Eosin for histological evaluation.

\section{Statistical Analysis}

Statistical analysis was performed using ANOVA and t-test in GraphPad Instant software (GraphPad Software, Inc.)

\section{Results}

\section{Fabrication and Characterization of nanostructured PCL-G scaffolds}


In this study, electrospun nanofibrous matrices of 40-60 $\mu \mathrm{m}$ thickness (Fig. S1a-d) with varying concentrations of graphene $(0.1 \mathrm{mg} / \mathrm{ml}, 0.5 \mathrm{mg} / \mathrm{ml}$, and $1 \mathrm{mg} / \mathrm{ml})$ (Fig. S1 e) were prepared. The diameter of the fibers in the scaffolds was in a range of $200 \mathrm{~nm}$ to $2 \mu \mathrm{m}$. The distributions of diameters for the samples are shown in Fig. 1a, b, along with their SEM images. The fibers were randomly oriented to form multiple layers resulting in a 3D nano-fibrous mat like scaffolds. Varying the graphene concentrations did not have any significant effect on nanofiber diameter as well as on the thickness of the scaffolds. The dispersion of graphene in the nanofiber was observed using TEM (Fig. S1f,g) and optical microscope (Fig. S1h,i) wherein graphene was seen to be successfully incorporated into the polymeric fibers. The presence of graphene in the fibers was also validated through SEM; protrusions were seen on the outer surface of PCL-G nanocomposites (Fig. S1j,k). PCL is a hydrophobic polymer that makes it difficult for cells to attach. Graphene has been reported to have high surface area and roughness, which improves cell adhesion to the hydrophobic polymer surfaces. The dispersion of graphene into PCL nanofibers renders the surface of the nanofibers hydrophilic in a concentration-dependent manner. Figure 1c represents the water contact angle measured on the surface of the PCL and PCL-G scaffolds. PCL-G0.01 did not show any significant change in the contact angle $\left(128^{\circ}\right)$ as compared to native PCL $\left(124^{\circ}\right)$ and PCL-G0.05 $\left(124^{\circ}\right)$. However, the contact angle decreased significantly by further increasing the concentration of graphene, PCL-G0.1 $\left(96^{\circ}\right)$, thus increasing the wettability of the scaffold $(p<0.0001)$. Furthermore, surface topography and roughness of PCL and PCL-G0.05 nanocomposite scaffolds were analyzed by AFM (Fig. 1d). The average roughness ( $\mathrm{Ra}$ ) and roughness mean square (Rms) of PCL nanofibres was $79.08 \mathrm{~nm}$ and $108.11 \mathrm{~nm}$, respectively. The surface roughness of the samples increased drastically by the addition of graphene, with PCL-G0.05 showing Ra and Rms values of $213.81 \mathrm{~nm}$ and $265.73 \mathrm{~nm}$, respectively. The presence of graphene in PCL-G nanocomposite scaffolds was evaluated by FTIR spectroscopy and XRD (Fig. S2a and b). There was no significant change in the FTIR spectra and XRD pattern of PCL-G nanocomposites w.r.t PCL, due to the very low concentrations of graphene used. The dispersion of graphene in the PCL-G nanocomposites was confirmed using Raman spectroscopy (Fig. S2C). The Raman spectra of graphene presented the peaks at $1348 \mathrm{~cm}^{-1}$ (D band), $1589 \mathrm{~cm}^{-1}$ (G band), and a sharp peak at $2703 \mathrm{~cm}-1$ (2D band). In pure PCL scaffold, peaks at $2913 \mathrm{~cm}^{-1}, 1723 \mathrm{~cm}-1$ corresponding to $\mathrm{C}-\mathrm{H}$, and $\mathrm{C}=\mathrm{O}$ stretching vibrations were observed. The $\mathrm{C}-\mathrm{H}$ bending vibrations observed at $1441 \mathrm{~cm}^{-1}$ in the PCL scaffold was enhanced in the case of PCL-G0.05 and PCL-G0.1 nanocomposite scaffold. Additionally, the peak corresponding to 2D band of graphene was observed to shift to around $2740 \mathrm{~cm}^{-1}$ in PCL-G0.05 and PCL-G0.1 nanocomposite scaffolds confirming the presence of graphene in the scaffolds and strong interactions of graphene with the PCL matrices. There were no significant changes in the Raman spectrum of PCL-G0.01 as compared to PCL due to a very low amount of graphene in it. Thermal stability and degradation behavior of PCL and PCL-G nanocomposite scaffolds were studied using TGA (Fig. S3a). No significant differences were seen in the thermal degradation profiles of the PCL and PCL-G nanocomposites scaffolds. Their onset degradation temperature started from $350^{\circ} \mathrm{C}$ and continued up to $450^{\circ} \mathrm{C}$. The weight loss after $450^{\circ} \mathrm{C}$ was negligible; there was an increase in char formation by $1-2 \%$ after the addition of $\mathrm{G}$ in the composite scaffolds. The melting behavior of PCL and PCL-G nanocomposite scaffolds was evaluated by DSC (Fig. S3b). The melting point of the pure PCL scaffold was $60.1^{\circ} \mathrm{C}$, which decreased to $55.4^{\circ} \mathrm{C}$ for PCL-G0.05 and to $57.6{ }^{\circ} \mathrm{C}$ for 
PCL-G0.1. The decrease in the melting point upon the addition of graphene could be due to the interaction of graphene with PCL, which may prevent the formation of defect-free crystalline phase in PCL. The mechanical strength of the scaffolds was determined by measuring load as a function of extension. Figure. S3c represents a plot between the load versus the extension applied. The overall deformation behaviour of the PCL-G nanocomposite scaffolds was similar to the pure PCL scaffold. However, the tensile strength of the PCL-G nanocomposites changed with the addition of $\mathrm{G}$. The tensile strength of the PCL scaffold was $0.66 \mathrm{MPa}$ which increased to $0.71 \mathrm{MPa}$ for PCL-G0.01, which further increased to 1.25 MPa for PCL-G0.05, and then decreased to 1.1 MPa for PCL-G0.1. This could be due to good dispersion of the graphene in the PCL polymer matrix for PCL-G0.05. Increasing the concentration of graphene to $0.1 \%$ may lead to their agglomeration resulting in decrease in the tensile strength. The higher concentration of graphene facilitates strong $\pi-\pi$ interactions among the stacked graphene sheets causing them to agglomerate, thereby affecting their dispersion in the polymer matrices. The Young's modulus, which is a measure of the stiffness of a material, also increased on the addition of graphene to the PCL matrix (Fig. S3d). Figure. S4 e, f, show plots of shear storage ( $\left.G^{\prime}\right)$ and loss ( $\left.G^{\prime \prime}\right)$ modulus versus the oscillatory frequency $(\omega)$, respectively. $G^{\prime}(\omega)>G^{\prime \prime}(\omega)$ is seen in all the composites indicating frequency independence and a stable elastic gel-like behavior. Also, the $G^{\prime}$ decreases with increasing concentration of graphene in PCL; the reduction was significant for PCL-G0.1 and PCL-G0.05 only.

\section{Biocompatibility Of Electrospun Nanofibrous Scaffolds}

The nature of the scaffold dictates the behaviour of cells in terms of their viability, attachment, morphology, proliferation, cell signalling, Extracellular Matrix (ECM) secretion, and differentiation. Figure. 3 , represents hMSCs response to PCL surface in the absence or presence of different concentrations of graphene assessing the above parameters. In all the studies, nanocomposite was compared to a conventional cover glass surface (control). PCL and PCL-G nanocomposites cytotoxicity was evaluated by Calcein-AM and EthD-I staining assay (Fig. 2a-e). Almost $90 \%$ of the cells cultured on control, PCL and all PCL-G nanocomposite were viable. Cellular morphology of hMSCs cultured over PCL and PCL-G nanocomposites was evaluated by studying the SEM images.

Majority of stem cells were seen to exhibit typical spindle-shaped morphology and were well attached, elongated, and evenly spread on all PCL and PCL-G nanocomposite (Fig. 2f-j). Additionally, the secreted ECM was assessed by Collagen-I (Col-I) at day 7, with PCL-G0.05 and PCL-G0.1 giving best results (Fig. 3a-o). The relative expression of Collagen-I secreted by PCL-G nanocomposite, also showed no significant difference (Figure S5). hMSCs proliferation on the coverslip, PCL, and PCL-G nanocomposites mats was examined by measuring the DNA content level by PI/RNase staining and quantification was done on 1st, 3rd and 5th day. At day 1, there was no significant difference in DNA content between the PCL and PCL-G nanocomposites, however, the DNA content increased in all variants from day 3 onwards, 
indicating robust proliferation in all the studied groups (Fig. S6a,b). To test whether the basal mitochondrial respiration varies among MSCs grown on the cover glass surface and PCL nanocomposites, we measured basal mitochondrial respiration rate. No significant difference in mitochondrial respiration was found among all the groups, indicating good bioenergetics of the cell metabolic state (Fig. S7). Any influence on the bioenergetic pathway decisively affects the genetic stability of the human stem cell ${ }^{29}$. To assess the In vivo biocompatibility of the as-synthesized nanocomposites, we performed the incision on the dorsal side of Wistar rats with an area of approx. $1 \times$ $1 \mathrm{~cm}$ and implanted the nanomembranes for 15 days. H\&E was performed to observe the histopathology of the incised wound area and showed no significant difference in the appearance of the incised area when compared to control and PCL-G nanocomposites. Neither any residue of the implanted membranes nor any significant inflammation was seen at the site of injury. Very few inflammatory cells and Fibroblasts were seen at higher magnification of PCL-G nanocomposite groups as compared to control. Natural healing phase of blood vessels was observed with no granuloma and foreign-body giant cell reaction secondary to the implanted membranes.

\section{Stem Cell Differentiation Into Da Neurons On Pcl-g Nanocomposites}

Differentiation potential of hMSCs towards DA neurons on the as-synthesized PCL-G nanocomposites was examined by immunofluorescence assay using specific markers such as MAP-2, Beta-III-tubulin, and TH. Expression of MAP-2 and TH indicates the neuronal differentiation of hMSCs, whereas, TH expression confirms the presence of DA neurons (Fig. 3a-j). The Mean Fluorescent Intensity (MFI) of MAP-2 (Fig. 3k) and TH (Fig. 3l) was significantly high in cells grown on PCL-G0.05 and PCL-G0.1 as compared to control and PCL only, the same trend was observed in the Beta-III tubulin expression (Fig. S8a-k). We utilized PCL-G nanocomposites scaffolds as a stimulator to enhance the differentiation of hMSCs into neurons. Interestingly, it was observed that the expression of both neuronal \& DA markers (MAP-2 and TH respectively) was significantly high in the un-induced groups as well, across all the nanocomposites when compared to coverslip and PCL only hinting towards the inherent capability of the graphene-based nanocomposites to coax hMSCs into neuronal differentiation. Morphology of differentiated neurons grown over the PCL, and PCL-G nanocomposites was examined by SEM (Fig. 4a). After 14 days of culture, a significant difference in the cellular morphology was evident on PCL and PCL$\mathrm{G}$ nanocomposites compared to the un-induced group. PCL-G nanocomposites displayed large neurite to cell body ratio, long neurite processes, their wrapping around the nanofiber, and multiple outgrowths coming out of the cell body (Fig. S9, a-c). The above observations support the hypothesis that the assynthesized nanocomposite aids in the neuronal differentiation of hMSCs. One of the hallmark features of neuronal differentiation is the neurite outgrowth. It was observed that the majority of stem cells underwent morphological changes when incubated in differentiation media. Therefore, we investigated the possible role of nanocomposite in the neurite extension. Figure. $4 \mathrm{~b}$ displays a positive effect on the neurite length $(100-150 \mu \mathrm{m})$ of cells grown on high graphene concentration as compared to lower 
concentration giving only $~ 50 \mu \mathrm{m}$. Numerous neurites were found with $150 \mu \mathrm{m}$ length in PCL-G0.05 and PCL-G0.01 nanocomposites. To study the relative impact of contact guidance on neurite length outgrowth, we investigated whether the electrospun mess organization affects the cells orientation or/and neuronal network.

It was seen that the cytoskeleton arrangement of the cells grown over the electrospun scaffolds, was influenced by the concentration of graphene in the nanocomposites that plays a major role in determining cell morphology, differentiation, and orientation (Fig. 5a-d). The maximum parallel alignment was seen in hMSCs grown over PCL-G0.05 and PCL-G0.1 scaffold in comparison to other study groups. We subsequently quantified the mean orientation of the neuronal cells over the PCL and PCL-G nanocomposites. The high concentration of graphene resulted in better cell alignment as compared to the low concentrations. The Slice_1R, and Slice_1B represent the fluorescence of F-actin and DAPI stained cells, respectively. The total number of cells with combined directionality of all two slices was absent for all un-induced samples. In contrast, $70 \%$ of F-actin stained cells were found highest with a preferred orientation at $38.29^{\circ}$ for induced PCL, and lowest, $49 \%$ was detected for induced PCL-G0.01 among all induced samples. The obtained number of cells have a significant orientation with an acceptable goodness score (near to 1). The highest peak fitted by a Gaussian function is exhibited by induced PCLG0.1, which represents the periodic nature of the histogram. Likewise, the direction at $57.30^{\circ}$ signifies the center of the gaussian, while dispersion at $7.24^{\circ}$ reports the standard deviation (SD) of the gaussian. Nevertheless, the amount 0.61 indicates the sum of the histogram from the center- standard deviation to center + standard deviation, divided by the total sum of the histogram. The highest goodness score, 0.98 , noted for induced PCL-G0.1 confirms the significance of the obtained number of cells having orientation

(Fig. 6). Thus, according to observed results, the concentration of the graphene is critical in determining cell morphology and differentiation efficiency in vitro.

To understand the functional behaviour of newly differentiated neurons from hMSCs, we performed ELISA for dopamine release and Calcium ion imaging for intracellular $\mathrm{Ca}^{2+}$ influx. The functional characterization of the differentiated neurons was performed by estimating the dopamine released in the culture supernatant upon stimulation with $\mathrm{KCl}(56 \mathrm{mM})$. hMSCs differentiated into DA neurons secreted dopamine constitutively. The dopamine release from hMSCs cultured over PCL-G nanocomposites was measured for both un-induced and induced hMSCs on day 14. As represented in Figure. 7a, the constitutive release of dopamine at day 14 was $840 \pm 41.63 \mathrm{pg} / \mathrm{ml}$, and the dopamine secreted upon the addition of $\mathrm{KCl}$ was $1050 \pm 100 \mathrm{pg} / \mathrm{ml}$. Control cells were maintained in regular culture medium and did not show any appreciable release of dopamine. Upon stimulation with $\mathrm{KCl}$, the intracellular $\mathrm{Ca}^{2+}$ influx and dopamine release were enhanced significantly, suggesting the presence of purinergic receptors and 
potassium ion channels in the induced cells. The cells were stained with a Fura-2-AM dye to monitor the change of intracellular calcium ion concentrations. The amount of fluorescence intensity changed by the $\mathrm{KCl}$ stimuli varied from cell to cell. Presumably, it is because individual cells had different interactions with the PCL-G0.1 and PCL-G0.05 surface, and not all were fully differentiated neurons, thereby giving varied response to external stimuli. Previous reports showed that the voltage pulse stimuli on a neuron from an electrode opened calcium ion channels and increased the calcium ion concentration in the neuron, resulting in the enhanced fluorescence intensity of Fura-2-AM dye in the neuron ${ }^{30}$. The cells exhibited an increased fluorescence intensity by $60-70 \%$ due to the electrical stimuli (Fig. $7 \mathrm{~b}$ ), with PCLG0.05 giving the best result, thereby confirming that the differentiated cells from hMSCs were functioning as a neuron (Multimedia file attached).

\section{Md Simulations Assessment Of Graphene And F-actin Interaction}

The modelled $G$ sheet was visualized and seemed to hold a sufficient surface area to bind (Fig. 8a). It was observed that $6 \mathrm{ANU}$ attained best resolution, which has a maximum sequence length of monomer $\mathrm{F}$ actin protein (Table 2). The F-actin was successfully docked and displayed a higher binding affinity, $-36 \mathrm{kcal} / \mathrm{mol}$ for graphene among all docked complexes. The independent simulation to test the structural stability of the F-actin in pure water is presented in Fig. 8b. The RMSD of the F-actin backbone fluctuated approximately at $3 \AA$, which describes the secondary structure of protein remained well in the water. Similarly, stabilized RMSD of graphene attained a plateau at $2.5 \AA$. Collectively, RMSD of F-actin and graphene shows that no deformation or broken structure was observed throughout the simulations. However, the RMSD had no distinct variation but fluctuated slightly until the simulation was finished, implying that the conformation of F-actin on the graphene surface was highly stable. The adsorption was driven by the intense attraction between F-actin and graphene, which could be depicted by the average Coulomb short-range (SR) interaction, $-1.53288 \mathrm{e}+06 \mathrm{~kJ} / \mathrm{mol}$ which was constantly maintained till the end of the simulation (Fig. 8c). This vdW interaction energy was corroborated by examining the Potential energy, $-1.10362 \mathrm{e}+06 \mathrm{~kJ} / \mathrm{mol}$; and Total energy, $-867267 \mathrm{~kJ} / \mathrm{mol}$, which was found persistent. This result exhibits that the strong interaction was established between F-actin and graphene, which was not disturbed due to simulations.

The minimum distance between F-actin and graphene was observed to be $2.4 \AA$ during the simulations, confirming a close contact between the two (Fig. 8d). This also established that F-actin was tightly adsorbed on the surface of graphene. It was found that there was 1250 number of contacts established between them within $4 \AA$ (Fig. 8e), indicating significant and stable interactions between the two. The higher number of these close contacts substantiate the stable interaction between F-actin and graphene. The geometries for adsorption were further optimized in the presence of a large number of water 
molecules showing only minor local changes. Therefore, the molecular size of the F-actin and graphene is unaffected by hydration, identified by both the radius of gyration and the principal axes (Fig. 8f).

Table 2: F-actin protein displays the PDB ID with method of 3D structure elucidation, resolution, and information of chains, sequence length and reason to choose the 3D structure which was used for docking.

\begin{tabular}{|c|c|c|c|c|c|}
\hline $\begin{array}{l}\text { PDB } \\
\text { entry }\end{array}$ & Method & $\begin{array}{l}\text { Resolution } \\
(\AA)\end{array}$ & Chain & Positions & $\begin{array}{l}\text { Reason to choose } \\
\text { for simulations }\end{array}$ \\
\hline 3BYH & $\begin{array}{l}\text { Electron } \\
\text { microscopy }\end{array}$ & 12.00 & $\mathrm{~A}$ & $2-375$ & $\begin{array}{l}\text { Facing problem } \\
\text { while performing } \\
\text { the equilibration } \\
\text { step }\end{array}$ \\
\hline $3 \mathrm{D} 2 \mathrm{U}$ & X-ray & 2.21 & $\mathrm{C} / \mathrm{G}$ & $170-178$ & $\begin{array}{l}\text { Do not have whole } \\
\text { structure }\end{array}$ \\
\hline $3 \mathrm{~J} 82$ & $\begin{array}{l}\text { electron } \\
\text { microscopy }\end{array}$ & 7.70 & $\mathrm{~B} / \mathrm{C} / \mathrm{D}$ & $2-375$ & $\begin{array}{l}\text { We can proceed } \\
\text { with this structure } \\
\text { but have lower } \\
\text { resolution than } \\
6 \mathrm{ANU}\end{array}$ \\
\hline 3LUE & $\begin{array}{l}\text { electron } \\
\text { microscopy }\end{array}$ & 15.00 & $\begin{array}{l}\mathrm{A} / \mathrm{B} / \mathrm{C} / \mathrm{D} / \\
\mathrm{E} / \mathrm{F} / \mathrm{G} / \mathrm{H} / \\
\mathrm{I} / \mathrm{J}\end{array}$ & $2-375$ & Poor resolution \\
\hline 6ANU & $\begin{array}{l}\text { electron } \\
\text { microscopy }\end{array}$ & 7.00 & $\begin{array}{l}\mathrm{A} / \mathrm{B} / \mathrm{C} / \mathrm{D} / \\
\mathrm{E} / \mathrm{F}\end{array}$ & $1-375$ & Best resolution \\
\hline 6ICT & X-ray & 1.95 & $\mathrm{E} / \mathrm{G} / \mathrm{H} / \mathrm{I}$ & $66-88$ & 23 aa only \\
\hline $6 \mathrm{ICV}$ & $\mathrm{X}$-ray & 2.15 & $\mathrm{C} / \mathrm{D}$ & $66-88$ & $23 \mathrm{aa}$ \\
\hline $6 \mathrm{MBJ}$ & $\mathrm{X}$-ray & 1.78 & $\mathrm{Y} / \mathrm{Z}$ & $66-80$ & 15 aа \\
\hline $6 \mathrm{MBK}$ & $\mathrm{X}$-ray & 1.69 & $\mathrm{Y} / \mathrm{Z}$ & $66-80$ & $15 \mathrm{aa}$ \\
\hline $6 \mathrm{MBL}$ & $\mathrm{X}$-ray & 2.20 & $\mathrm{Y}$ & $66-80$ & 15 aa \\
\hline
\end{tabular}

Conclusively, the conformational dynamics of the F-actin segment adsorption onto graphene was observed by the RMSD, which shows that the F-actin is well simulated with graphene within $100 \mathrm{~ns}$ of simulation time. This phenomenon can be attributed to the stabilization of the radius of gyration, Gromacs energies, and minimum distance and close contacts of F-actin due to its adsorption onto graphene, leading to maintenance of complex interactions. These results indicated that the F-actin and graphene complex stabilized at the end of simulations signifying their strong binding.

\section{Discussion}


In the present study, we fabricated highly porous electrospun PCL-G nanocomposite mats, opening a vista for the exchange of nutrients, oxygen and metabolic waste due to their high surface area-to-volume ratio which is meant to be ideal for tissue engineering applications. Over the years, research on graphene and its derivative has been among the fastest rising field in nanoscience and nanotechnology. Fundamentally, graphene is an allotrope of carbon, which comprises a single atom layer of six-carbon rings in a $2 \mathrm{D}$ honeycomb lattice network. Graphene has rare properties such as large specific surface area, robust surface chemistry, superior electrical conductivity, excellent thermal conductivity, and unparalleled mechanical strength [31, 32]. Therefore, the electrospinning of PCL with graphene is nearly an ideal 3D fabricated, randomly distributed fibrous structured scaffold that mimics the native microenvironment of many cells. The high surface area provided by nanofibrous scaffolds leads to an increase in cellular attachment and better cell polarity compared to that of other non-fibrillar surfaces. Furthermore, both Cukierman and Griffith individually have reported that 2D cultured cells may differ in morphology and differentiation patterns from those cultured in 3D structured topography [33, 34]. It has been reported that the 2D plane decreases the rate of attachment, proliferation, and differentiation over time due to restraint space for cell-cell interaction, whereas 3D planes with graphene have shown an ideal substrate for stem cell differentiation [35]. To achieve better cell attachment, morphology, and differentiation, various studies have used different concentrations of graphene blended with different polymers (e.g. PCL, PLA, PET, Chitosan, Silk etc). Our fabricated PCL-G nanocomposites have shown the fiber diameter, ranging from $200 \mathrm{~nm}$ to $2 \mu \mathrm{m}$, irrespective of the graphene concentration, thereby mimicking the nano-to-micro scale pattern of the ECM. SEM and TEM micrographs revealed a homogeneous dispersion of graphene in the PCL nanofibers. PCL, in its native form, is hydrophobic, which makes it unfavourable for cell attachment. In a recent finding of Munz and colleagues, it was shown that the hydrophilicity of the nano-fibers surface increased with the addition of graphene [36], a same trend was observed for our PCL-G nanocomposite scaffolds. One of the major contributors towards this increase in hydrophilicity is the increase in surface roughness of the nanofibrous mats owing to the dispersion of graphene as revealed by the AFM studies which lies in accordance to Wenzel theoretical model [37], further supporting the contact angle findings. The addition of graphene not only modulated the topology but also enhanced the tensile strength of the nanocomposite over pure PCL. As the viscoelastic moduli of the nanocomposites were found to be frequency independent with Young's modulus ( $G$ ') higher than the viscous one (G"), they exhibited "strong gel" character. However, the increased concentration of graphene led to a decrease in the elastic modulus, indicating a strong association between the PCL chains with graphene, which is likely to decrease the entanglements among the PCL chains. This is in accordance with the report of Holmes et al. that naïve graphene interacts with polymer only through Van der Waals forces, $\pi-\pi$ stacking, and hydrophobic interactions [38]. However, graphene helps in reinforcement of PCL matrix resulting in better tensile properties of the scaffolds at low additions [39]. The facile electrospinning behaviour of nanocomposites was supported by the rheological studies showing a decrease in the G' values of PCL-G nanocomposites with an increase in graphene concentration. The increased stability of PCL-G nanocomposites was evident in weight loss profile. It was seen that the weight loss of pure PCL and PCL-G nanocomposite scaffolds was similar and appeared to be a monotonic weight loss that begins at approximately $350^{\circ} \mathrm{C}$ due to the decomposition of the PCL 
chains. The char formed increased after addition of graphene. A slight decrease in melting temperature of nanocomposite with increase in graphene was observed, which could be attributed to the formation of defective crystals of PCL resulting from restrictive movement of PCL chains in the presence of Graphene. Very low concentrations of graphene used as fillers $(0.01$, and 0.05$)$ did not produce any $D$ and $G$ bands in the PCL-G nanocomposites in Raman spectra. However, a small G- band hump and 2D band could be observed in PCL-G0.1 validating the successful incorporation of graphene and its strong interaction with PCL. The inherent toxicity of pristine graphene $[40,41]$ was evaded by intensively choosing the optimum mixing concentration of graphene and electrospinning it with PCL. This was confirmed by the cytocompatibility, cell attachment, proliferation, and mitochondrial OCR levels. These nanocomposites were highly cytocompatible with maximum cells seen live as assessed by calcein staining. Apart from higher cell attachment, PCL-G nanocomposites also favoured enhanced cell proliferation and better mitochondrial health, indicating good metabolic state as compared to pure PCL. In our study we also found that $0.05 \%$ graphene supported ECM secretion, keeping their morphology intact and stem cell differentiation. It is well known that cell-ECM interaction is one of the potent aspects in determining the fate of stem cells. Keeping this in mind, we used different concentrations of graphene, with PCL-0.05G giving better ECM secretion and stem cell differentiation. We noticed a concurrent upregulation of Col-I secretion by hMSCs with the increasing graphene concentration in the PCL nanocomposites, which indicates that the as-synthesized nanocomposites provide an excellent substrate for better cell attachment and survival. On the other hand, all the scaffolds were found to be histocompatible in animal model with no granuloma and foreign body giant cell reaction seen against the material.

Bestowed with excellent biocompatibility, PCL-G nanocomposites also demonstrated a remarkable potential to differentiate stem cells into neuronal cells. This observed phenomenon is due to a sophisticated interplay of chemical and physical properties of graphene nanocomposites and cells. However, we, for the first time, are reporting the differentiation of hMSCs into functional DA neurons, using nanocomposite scaffolds having the least possible concentration of graphene, thereby promoting their translational value for targeted treatment, such as Parkinson's disease. Therefore, we designed a one-step and economical strategy, devoid of any cross-linker to fabricate scaffolds that promotes the differentiation of hMSCs into DA neurons which were characterized by multi-parametric approaches. Comprehensive studies validated the role of PCL-G nanocomposites into enhanced differentiation and functionality of hMSCs into DA neurons. Some of the interesting features like Neurite length, directionality, cell orientation, ECM secretion and DA secretion were validated and were seen to be enhanced in the case of nanocomposite having least concentration of graphene (PCL-G0.05) which was therefore was considered to be the best. Another interesting observation in our study was the enhanced neuronal markers expression in the uninduced group. This is credited to the presence of graphene in PCL nanocomposites. It has been already observed that the use of low concentration of graphene has a strong modulatory effect of active and passive bioelectric properties on the cell membrane, which aids in differentiation of hMSCs with typical spindle-shaped morphology [42].The interplay of graphene and fine PCL nanofibers further enhanced neurite outgrowths with PCL-G0.05 and PCL-G0.1 producing remarkably longer neurites as compared to other as-synthesized scaffolds. Increasing concentrations of graphene 
yielded softer nanocomposites, which are neurogenic in nature, thereby aiding the generation of long neurites from hMSCs [43]. Additionally, the high electro-conductive property of graphene is also responsible for better neurite outgrowth $[44,45]$. More than any other cell type, neurons largely depend on the cytoskeleton, for their distinctive morphology, whose protein components are organized in a set of micro-differentiated compartments imitating the polarized form of the cell and play a significant role in determining its development [46]. One of the major changes occurring post differentiation is the rearrangement of microfilaments and microtubules. They both, on one hand, guides and supports the growth and differentiation of axons and dendrites; the dynamic actin filaments, on the other, drive the exploratory activity of growth cones as they respond to external guidance cues. Microtubules further stabilize the structure of the newly established processes [47]. Therefore, we examined the change in Factin arrangement among un-induced hMSCs and induced hMSCs to DA neurons on PCL-G nanocomposites. And we observed that graphene plays an important role in orienting the actin filaments of the cells undergoing differentiation over randomly aligned fibers [48]. Our PCL-G nanocomposite scaffold (PCL-G0.05 and PCL-G0.1) exhibited the best aligned cells, along with heightened neuronal expression. Although there are reports stating the enhanced differentiation of stem cells into neurons [49], ours goes a step ahead by harnessing the ability of human derived MSCs to differentiate into functional DA neurons. For better understanding the functionality of the generated neurons, we assayed the dopamine release and intracellular $\mathrm{Ca}^{2+}$ influx, which are the characteristic hallmark of mature functional neurons where, the neurotransmitter is stored in vesicles within the cell, and its release can be triggered by the influx of intracellular $\mathrm{Ca}^{2+}$. The rise in intracellular calcium ion influx in the neural circuitry upon excitation with $\mathrm{KCl}$ showed the functionality of neurons grown over nanocomposite[50]. Altogether, this establishes the synergistic ability of nano-fibers and graphene to not only have an impact on neurotic outgrowth but also orient the neurons to establish a neuronal network. The biological interactions between mammalian cell's F-actin and graphene are well documented [51], but mechanistic elucidation of this interaction is still a challenge. Intrigued by these finding and being aware that F-actin is the first respondent to external stimuli, we performed MD simulation to investigate the adhesive characteristics of F-actin-Graphene systems. For this, Gromacs energies, minimum distance and number of contacts established between F-actin and graphene, and compactness of the structures are criteria indicating the strength of protein adhesion, which is extensively applicable in tissue engineering to elucidate the effect of graphene affinity towards F-actin. The simulation results confirm that interactions between F-actin and graphene are much stronger. The conformation of F-actin on the graphene surface is highly stable via Coulomb short-range (SR) interaction, while potential and total energies confirmed steady complex. A comparison between experimental and simulation results suggested a meaningful correlation as well as strong interaction between F-actin and graphene. The results of this study provided a better understanding of the underlying mechanism for the interaction of hMSCs with graphene which is a necessary consideration for designing scaffolds.

\section{Conclusions}


In this work, we successfully demonstrated the ability of PCL-G nanocomposite scaffolds to efficiently differentiate hMSCs into DA neurons without using any additional growth factors. The facile, one-step electrospinning of PCL-G nanocomposite mats, as well as their easy handling and storage at room temperature, made the entire system very economical. The role of composition was meticulously considered for the cell-material interaction. For the best of our knowledge this is the first report harnessing the potential of graphene and PCL for the differentiation of human MSCs into functional DA neurons. Finally, herein reported, PCL-G nanocomposites can be used as excellent nanostructured neuronal prosthetics controlling hMSCs behaviour to probe their morphology and functionality opening a new horizon for an array of applications in neural regenerative medicine. However, the efficacy of these hMSCs differentiated DA neurons on PCL-G nanocomposites needs to be further validated In vivo.

\section{Abbreviations}

embryonic stem cells (ESCs), neural precursor cells (NPCs), or induced pluripotent stem cells (iPSCs), Mesenchymal Stem Cells (MSCs), Polylactic acid (PLA), poly(glycolic acid) (PGA), Polyethylene terephthalate (PET), polycaprolactone (PCL), Graphene (G), Dopaminergic (DA), graphene oxide (GO), carbon nanotubes (CNTs), Methylthiazolyldiphenyl-tetrazolium bromide (MTS), scanning electron microscopy (SEM), High-resolution transmission electron microscopy (HRTEM), transmission electron microscopy (TEM), Fourier transform Infrared spectroscopy (FTIR), X-ray diffraction (XRD), Atomic force microscopy (AFM), Thermogravimetric analysis (TGA), Differential scanning calorimetry (DSC), culture medium (CM), Oxygen consumption rate (OCR), Extracellular matrix (ECM), Hematoxylin \& Eosin (H\&E), Immunohistochemistry (IHC), Endothelial growth factor (EGF), Fibroblast growth factor (FGF-2), Microtubule Associated Protein (MAP-2), Tyrosine Hydroxylase (TH), Molecular dynamics (MD), Protein Data Bank (PDB), Optimized Protein Liquid Simulations (OPLS), Particle mesh Ewald (PME), Linear Constraint Solver (LINCS), Mean Fluorescent Intensity (MFI), Enzyme linked Immunosorbent Assay (ELISA), Short-Range (SR).

\section{Declarations}

\section{Author Contributions}

Krishan Gopal Jain prepared Scaffolds, acquired SEM images, and performed cell culture over scaffolds, ELISA assay. Sonali Rawat performed and analyzed Live/dead and IF staining, acquired images, Calcium ion flux imaging, and data interpretation. Deepika Gupta, Varun Arora and Adeeba Shakeel performed mechanical testing and physical characterization of the scaffolds (Raman, AFM, FTIR, Rheology, XRD, tensile and TEM) and data interpretation. Harshita Sharma expanded the mesenchymal stem cells. Pawan Raghav performed MD simulation studies. Ritupurna Chaudhuri performed Oxygen consumption rate experiment and analyzed mitochondria health. Debika Debnath and Ankarao Kalluri, gave inputs in scaffolds fabrication. Manjeet Jassal and Ashwini Agarwal provided tissue engineering supervision. Pinky carried out the animal studies and Amit Kumar Dinda helped in analysing the histopathological data. Sujata Mohanty and Prabir Patra provided advice on experimental design and data analysis. Sujata 
Mohanty designed the experiments, supervised the project and gave final approval of the manuscript. Sujata Mohanty, Sonali Rawat and Adeeba Shakeel wrote the manuscript.

\section{Acknowledgements}

The authors would like to thank All India Institute of Medical Sciences (AIIMS, New Delhi), for providing the research infrastructure. This work was supported by the Department of Biotechnology, Government of India (Grant no BT/01/COE/07/03). The authors would also like to thank Dr. Prabir Patra (University of Bridgeport, USA) for kindly providing the Graphene used in this study, Rudra for discussing XRD data, H.B Bohidar for Rheology data analysis, R. Pokle for SEM images colouring; Manisha Singh for inputs in Electron Microscopy and Mahak Tiwari for assisting in cell culture studies. Dr. Harpreet Singh, Indian Council for Medical Research (ICMR)-AIIMS computational genomics centre is acknowledged for supporting MD simulation studies and Delhi University for confocal microscopy, and Dr Sudhir Arav, Department of Pathology, AIIMS, New Delhi for his help and guidance in preparing the histopathological slides. All the data needed to evaluate the conclusions made in this paper are present within the data presented in the paper and/or the Supplemental Materials. Additional data may be requested from the authors.

\section{Ethics declarations}

Ethics approval and consent to participate

The present study was approved by the Institutional committee for Stem Cell Research, All India Institute of Medical Sciences, New Delhi, India. Informed consent was obtained from all participants, and experiments were conducted along the guidelines set by the Ethics Committee.

\section{Consent for publication}

Not applicable.

\section{Competing interests}

The authors indicated no potential conflicts of interest.

\section{Availability of data and material}

The data and material can be found with the corresponding author.

\section{Funding}

This work was funded by the Department of Biotechnology, Govt. of India (BT/01/COE/07/03) India. We are also grateful for the support of the All India Institute of Medical Sciences, New Delhi.

\section{References}


1. Komnig D, Imgrund S, Reich A, Gründer S, Falkenburger BH. ASIC1a deficient mice show unaltered neurodegeneration in the subacute MPTP model of Parkinson disease. PLoS One. 2016 Nov 7;11(11):e0165235.

2. Jankovic J. Parkinson's disease: clinical features and diagnosis. Journal of neurology, neurosurgery \& psychiatry. 2008 Apr 1;79(4):368 - 76.

3. Parmar M, Torper O, Drouin-Ouellet J. Cell-based therapy for Parkinson's disease: A journey through decades toward the light side of the Force. Eur J Neurosci. 2019 Feb;49(4):463-71.

4. Phinney DG, Isakova I. Plasticity and therapeutic potential of mesenchymal stem cells in the nervous system. Current pharmaceutical design. 2005 Apr 1;11(10):1255-65.

5. Barry FP, Murphy JM. Mesenchymal stem cells: clinical applications and biological characterization. The international journal of biochemistry \& cell biology. 2004 Apr 1;36(4):568 - 84.

6. Farini A, Sitzia C, Erratico S, Meregalli M, Torrente Y. Clinical applications of mesenchymal stem cells in chronic diseases. Stem cells international. 2014 Jan 1;2014.

7. Liu ZJ, Zhuge Y, Velazquez OC. Trafficking and differentiation of mesenchymal stem cells. Journal of cellular biochemistry. 2009 Apr 15;106(6):984-91.

8. Gomes M, Azevedo H, Malafaya P, Silva S, Oliveira J, Silva G, Sousa R, Mano J, Reis R. Natural polymers in tissue engineering applications. InTissue engineering 2008 Jan 1 (pp. 145-192). Academic Press.

9. Srikanth M, Asmatulu R, Cluff K, Yao L. Material characterization and bioanalysis of hybrid scaffolds of carbon nanomaterial and polymer nanofibers. ACS omega. 2019 Mar 8;4(3):5044-51.

10. Tiaw KS, Goh SW, Hong M, Wang Z, Lan B, Teoh SH. Laser surface modification of poly ( $\varepsilon$ caprolactone)(PCL) membrane for tissue engineering applications. Biomaterials. $2005 \mathrm{Mar}$ 1;26(7):763-9.

11. Yang K, Yu SJ, Lee JS, Lee HR, Chang GE, Seo J, Lee T, Cheong E, Im SG, Cho SW. Electroconductive nanoscale topography for enhanced neuronal differentiation and electrophysiological maturation of human neural stem cells. Nanoscale. 2017;9(47):18737-52.

12. Li N, Zhang Q, Gao S, Song Q, Huang R, Wang L, Liu L, Dai J, Tang M, Cheng G. Three-dimensional graphene foam as a biocompatible and conductive scaffold for neural stem cells. Scientific reports. 2013 Apr;3(1):1-6. 3(.

13. Kotov NA, Winter JO, Clements IP, Jan E, Timko BP, Campidelli S, Pathak S, Mazzatenta A, Lieber CM, Prato M, Bellamkonda RV. Nanomaterials for neural interfaces. Adv Mater. 2009 Oct;26(40):39704004. 21.

14. Bullock CJ, Bussy C. Biocompatibility considerations in the design of graphene biomedical materials. Advanced Materials Interfaces. 2019 Jun;6(11):1900229.

15. Park SY, Park J, Sim SH, Sung MG, Kim KS, Hong BH, Hong S. Enhanced differentiation of human neural stem cells into neurons on graphene. Advanced materials. 2011 Sep 22;23(36):H263-7. 
16. Lee WC, Lim CH, Shi H, Tang LA, Wang Y, Lim CT, Loh KP. Origin of enhanced stem cell growth and differentiation on graphene and graphene oxide. ACS Nano. 2011 Sep;27(9):7334-41. 5(.

17. Lee TJ, Park S, Bhang SH, Yoon JK, Jo I, Jeong GJ, Hong BH, Kim BS. Graphene enhances the cardiomyogenic differentiation of human embryonic stem cells. Biochemical and biophysical research communications. 2014 Sep 12;452(1):174 - 80.

18. Akhavan O, Ghaderi E, Shirazian SA, Rahighi R. Rolled graphene oxide foams as three-dimensional scaffolds for growth of neural fibers using electrical stimulation of stem cells. Carbon. 2016 Feb $1 ; 97: 71-7$.

19. Kumar S, Parekh SH. Linking graphene-based material physicochemical properties with molecular adsorption, structure and cell fate. Communications Chemistry. 2020 Jan 20;3(1):1-1.

20. Xia L, Zhu W, Wang Y, He S, Chai R. Regulation of neural stem cell proliferation and differentiation by graphene-based biomaterials. Neural plasticity. 2019 Oct 16;2019.

21. Palejwala AH, Fridley JS, Mata JA, Samuel EL, Luerssen TG, Perlaky L, Kent TA, Tour JM, Jea A. Biocompatibility of reduced graphene oxide nanoscaffolds following acute spinal cord injury in rats. Surgical neurology international. 2016;7.

22. Song J, Gao H, Zhu G, Cao X, Shi X, Wang Y. The preparation and characterization of polycaprolactone/graphene oxide biocomposite nanofiber scaffolds and their application for directing cell behaviors. Carbon. 2015 Dec;1:95:1039-50.

23. Ramazani S, Karimi M. Electrospinning of poly ( $\varepsilon$-caprolactone) solutions containing graphene oxide: Effects of graphene oxide content and oxidation level. Polymer Composites. 2016 Jan;37(1):131-40.

24. Lopresti F, Maio A, Botta L, Scaffaro R. Preparation and mechanical characterization of polycaprolactone/graphene oxide biocomposite nanofibers. InAIP Conference Proceedings 2016 May 18 (Vol. 1736, No. 1, p. 020105). AIP Publishing LLC.

25. Mohanta D, Patnaik S, Sood S, Das N. Carbon nanotubes: Evaluation of toxicity at biointerfaces. Journal of Pharmaceutical Analysis. 2019 Oct 1;9(5):293-300.

26. Ginestra P. Manufacturing of polycaprolactone-Graphene fibers for nerve tissue engineering. J Mech Behav Biomed Mater. 2019 Dec;1:100:103387.

27. Mohanty S, Bose S, Jain KG, Bhargava B, Airan B. TGFß1 contributes to cardiomyogenic-like differentiation of human bone marrow mesenchymal stem cells. Int J Cardiol. 2013 Feb;10(1):93-9. $163($.

28. Mohanty S, Jain KG, Nandy SB, Kakkar A, Kumar M, Dinda AK, Singh H, Ray A. Iron oxide labeling does not affect differentiation potential of human bone marrow mesenchymal stem cells exhibited by their differentiation into cardiac and neuronal cells. Molecular and cellular biochemistry. 2018 Nov 1;448(1-2):17-26.

29. Estrada JC, Albo C, Benguria A, Dopazo A, Lopez-Romero P, Carrera-Quintanar L, Roche E, Clemente EP, Enriquez JA, Bernad A, Samper E. Culture of human mesenchymal stem cells at low oxygen 
tension improves growth and genetic stability by activating glycolysis. Cell Death Differentiation. 2012 May;19(5):743-55.

30. Barreto-Chang OL, Dolmetsch RE. Calcium imaging of cortical neurons using Fura-2 AM. JoVE (Journal of Visualized Experiments). 2009 Jan 19(23):e1067.

31. Kim TH, Lee T, El-Said WA, Choi JW. Graphene-based materials for stem cell applications. Materials. 2015 Dec;8(12):8674-90.

32. Guazzo R, Gardin C, Bellin G, Sbricoli L, Ferroni L, Ludovichetti FS, Piattelli A, Antoniac I, Bressan E, Zavan B. Graphene-based nanomaterials for tissue engineering in the dental field. Nanomaterials. 2018 May;8(5):349.

33. Cukierman E, Pankov R, Yamada KM. Cell interactions with three-dimensional matrices. Current opinion in cell biology. 2002 Oct 1;14(5):633 - 40.

34. Griffith LG, Swartz MA. Capturing complex 3D tissue physiology in vitro. Nature reviews Molecular cell biology. 2006 Mar;7(3):211-24.

35. Lee WC, Loh KP, Lim CT. When stem cells meet graphene: Opportunities and challenges in regenerative medicine. Biomaterials. 2018 Feb 1;155:236 - 50.

36. Munz M, Giusca CE, Myers-Ward RL, Gaskill DK, Kazakova O. Thickness-dependent hydrophobicity of epitaxial graphene. Acs Nano. 2015 Aug 25;9(8):8401-11.

37. Bundgaard H, Larsen JD. N-Sulfonyl imidates as a novel prodrug form for an ester function or a sulfonamide group. Journal of medicinal chemistry. 1988 Nov;31(11):2066-9.

38. Holmes B, Fang X, Zarate A, Keidar M, Zhang LG. Enhanced human bone marrow mesenchymal stem cell chondrogenic differentiation in electrospun constructs with carbon nanomaterials. Carbon. 2016 Feb;1:97:1-3.

39. Scaffaro R, Lopresti F, Maio A, Botta L, Rigogliuso S, Ghersi G. Electrospun PCL/GO-g-PEG structures: Processing-morphology-properties relationships. Compos Part A: Appl Sci Manufac. 2017 Jan;1:92:97-107.

40. Liao KH, Lin YS, Macosko CW, Haynes CL. Cytotoxicity of graphene oxide and graphene in human erythrocytes and skin fibroblasts. ACS applied materials \& interfaces. 2011 Jul 27;3(7):2607-15.

41. Chowdhury SM, Lalwani G, Zhang K, Yang JY, Neville K, Sitharaman B. Cell specific cytotoxicity and uptake of graphene nanoribbons. Biomaterials. 2013 Jan 1;34(1):283 - 93.

42. Guo R, Zhang S, Xiao M, Qian F, He Z, Li D, Zhang X, Li H, Yang X, Wang M, Chai R. Accelerating bioelectric functional development of neural stem cells by graphene coupling: implications for neural interfacing with conductive materials. Biomaterials. 2016 Nov 1;106:193-204.

43. Engler AJ, Sen S, Sweeney HL, Discher DE. Matrix elasticity directs stem cell lineage specification. Cell. 2006 Aug 25;126(4):677-89.

44. Shin SR, Li YC, Jang HL, Khoshakhlagh P, Akbari M, Nasajpour A, Zhang YS, Tamayol A, Khademhosseini A. Graphene-based materials for tissue engineering. Advanced drug delivery reviews. 2016 Oct 1;105:255 - 74. 
45. Bouzid T, Sinitskii A, Lim JY. Graphene platform for neural regenerative medicine. Neural regeneration research. 2016 Jun;11(6):894.

46. Mitchel JA, Hoffman-Kim D. Cellular scale anisotropic topography guides Schwann cell motility. PloS one. 2011 Sep;20(9):e24316. 6(.

47. Kapitein LC, Hoogenraad CC. Building the neuronal microtubule cytoskeleton. Neuron. 2015 Aug 5;87(3):492-506.

48. Liu C, Zhu C, Li J, Zhou P, Chen M, Yang H, Li B. The effect of the fibre orientation of electrospun scaffolds on the matrix production of rabbit annulus fibrosus-derived stem cells. Bone research. 2015 Jun;9:3:15012.

49. Aydin T, Gurcan C, Taheri H, Yilmazer A. Graphene Based Materials in Neural Tissue Regeneration. InCell Biology and Translational Medicine, Volume 32018 (pp. 129-142). Springer, Cham.

50. Rong G, Kim EH, Poskanzer KE, Clark HA. A method for estimating intracellular ion concentration using optical nanosensors and ratiometric imaging. Scientific reports. 2017 Sep 7;7(1):1-0.

51. Zhang B, Wei P, Zhou Z, Wei T. Interactions of graphene with mammalian cells: Molecular mechanisms and biomedical insights. Advanced drug delivery reviews. 2016 Oct 1;105:145 - 62.

\section{Figures}




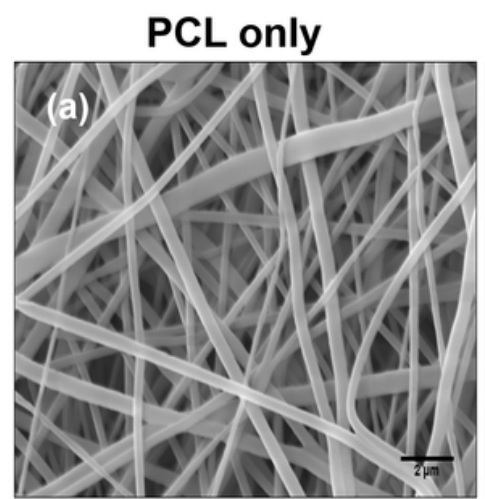

(b) Fiber Diameter Range in PCL Scaffold
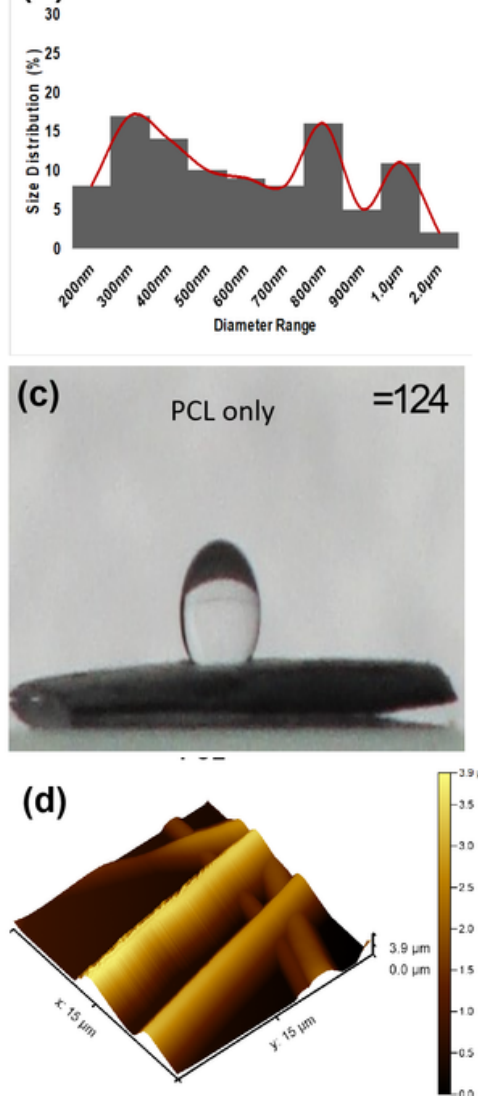

PCL-G0.01

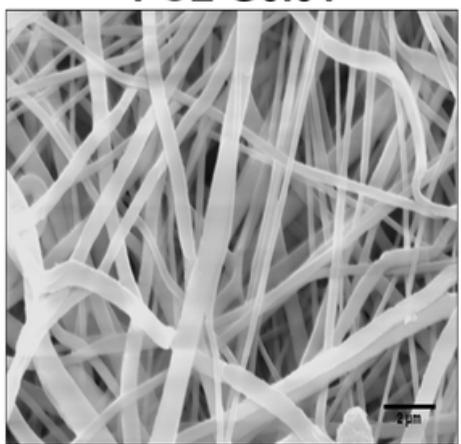

Fiber Diameter range in PCL-G0.01
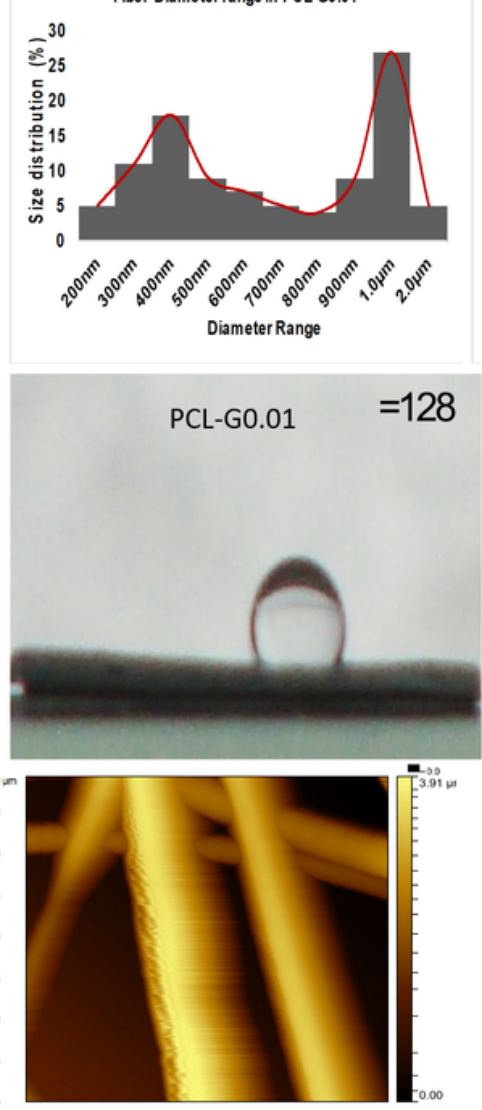

PCL-G0.05

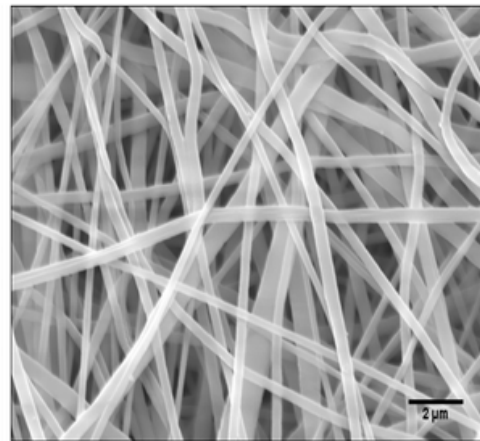

Fiber Diameter range in PCL-G0.05
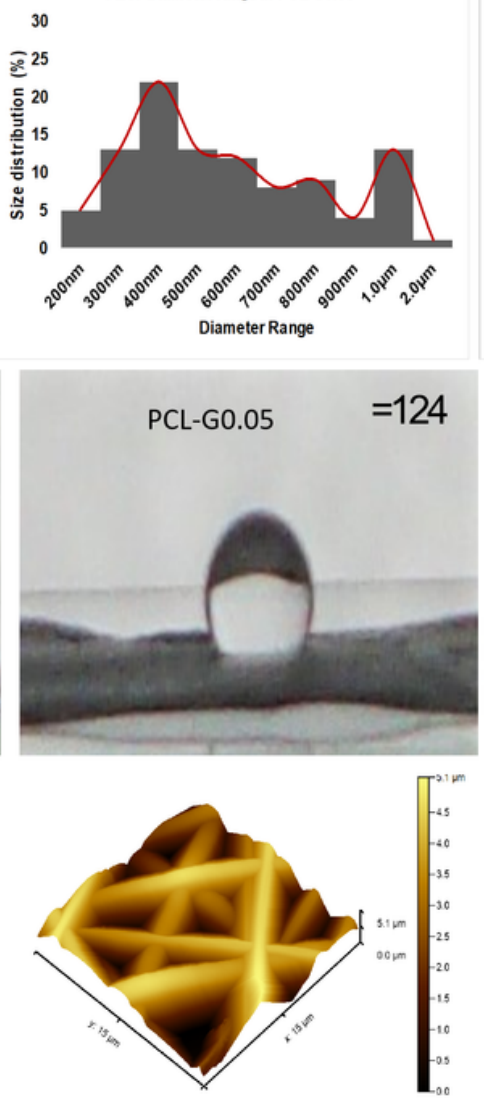

PCL-G0.1

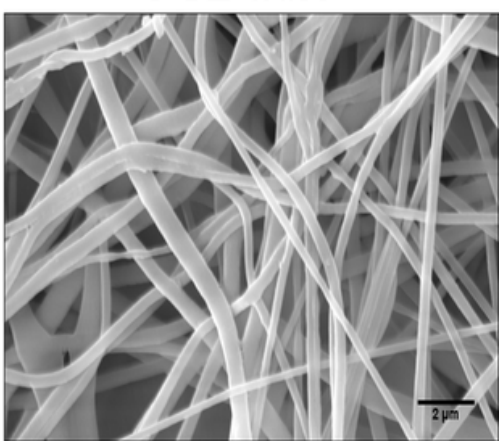

Fiber Diameter range in PCL-G0.1
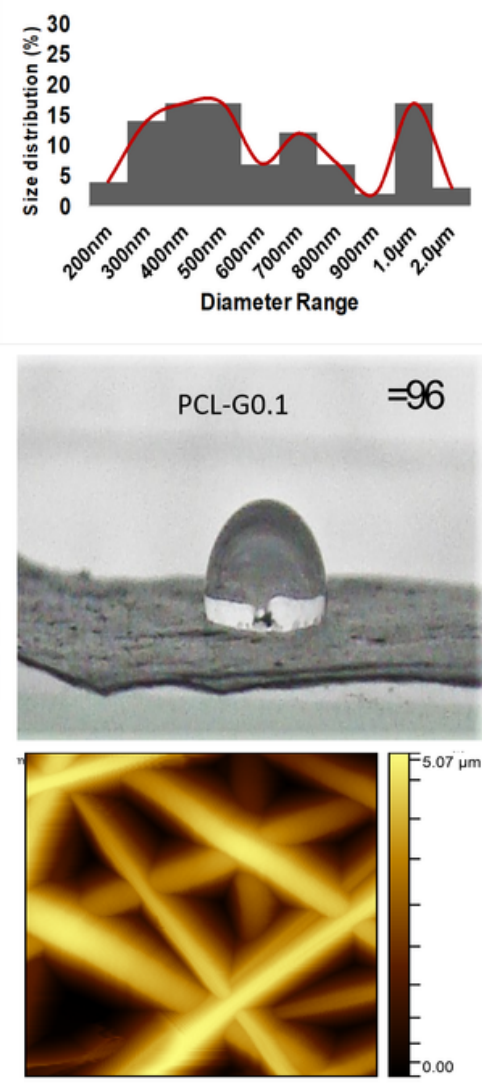

Figure 1

Characterisation of nanostructured PCL-G nanocomposite; (A,B) SEM images of electrospun nanofiber with average diameter ranging from 200-2000 $\mathrm{nm}$. (C) Contact angle represents the difference in wettability of PCL versus PCL-G nanocomposite, which indicates that increase in G concentration increases the wettability of the scaffold (D) AFM analysis revealed the average surface roughness of PCL and PCL-G0.05 nanocomposite showing an increase in the surface roughness of PCL with $G$ incorporation. 


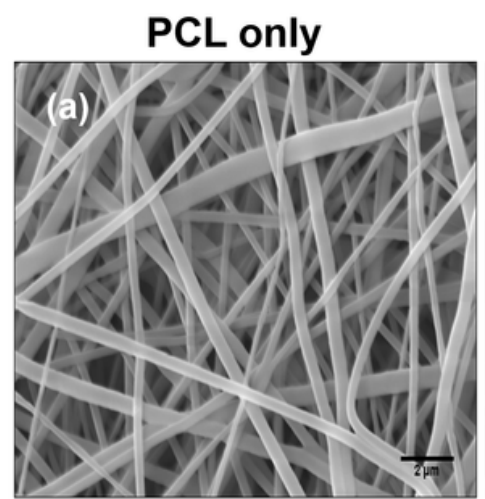

(b) Fiber Diameter Range in PCL Scaffold
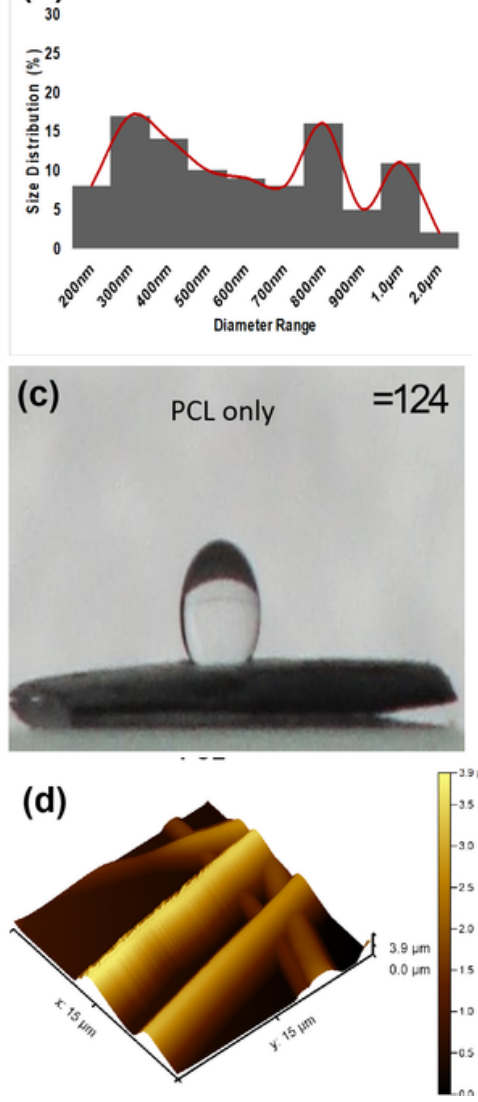

PCL-G0.01

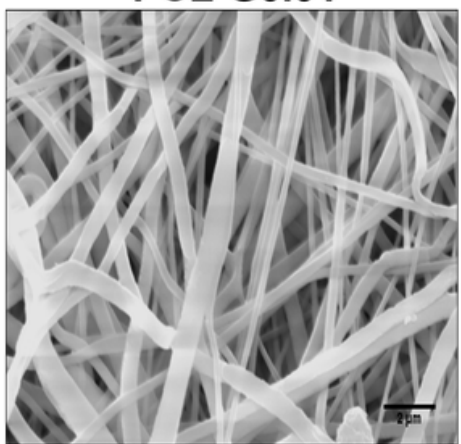

Fiber Diameter range in PCL-G0.01
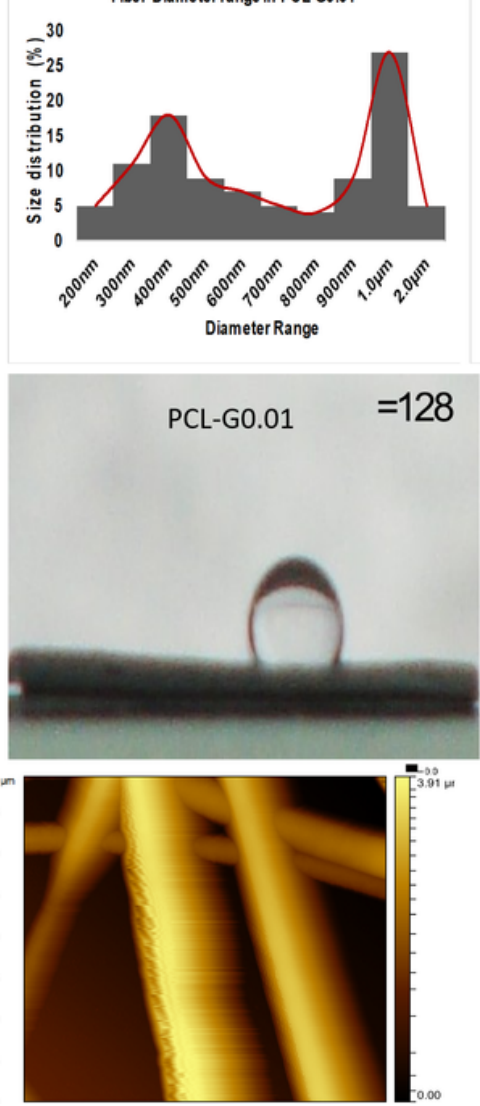

PCL-G0.05

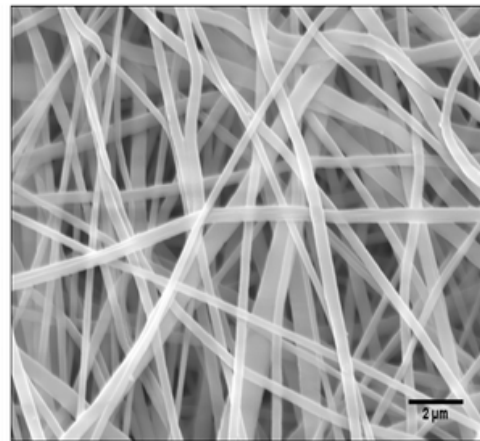

Fiber Diameter range in PCL-G0.05
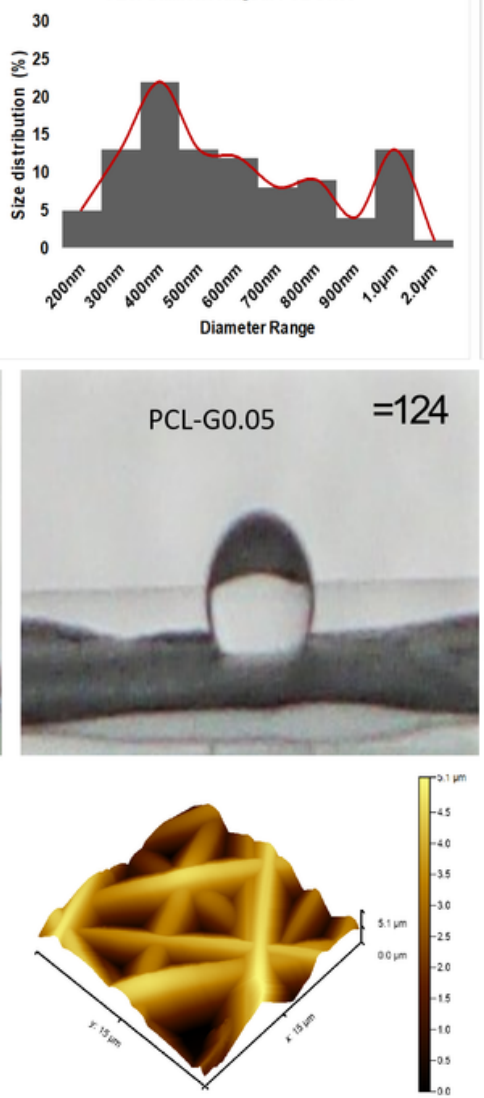

PCL-G0.1

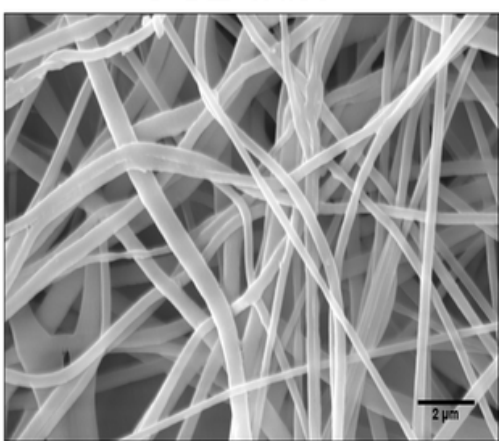

Fiber Diameter range in PCL-G0.1
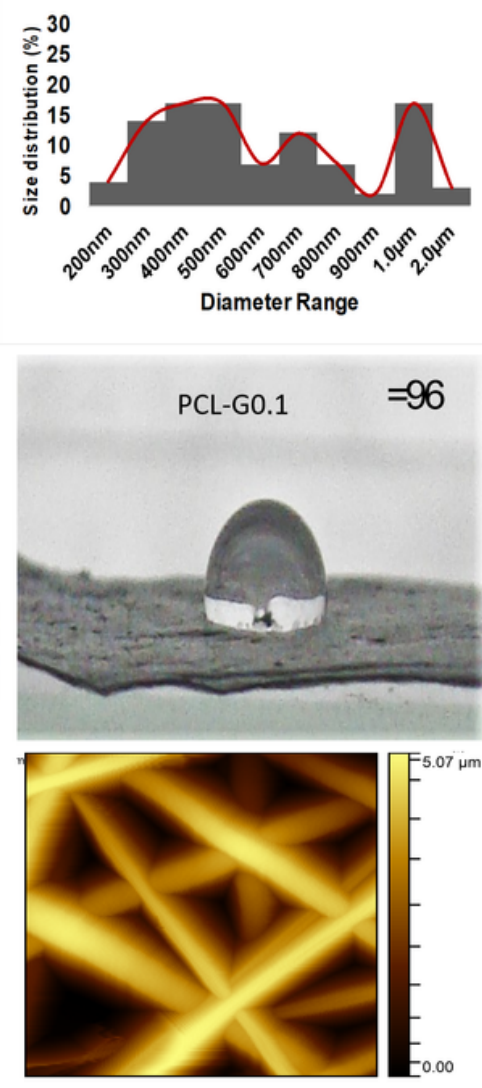

Figure 1

Characterisation of nanostructured PCL-G nanocomposite; (A,B) SEM images of electrospun nanofiber with average diameter ranging from 200-2000 $\mathrm{nm}$. (C) Contact angle represents the difference in wettability of PCL versus PCL-G nanocomposite, which indicates that increase in $\mathrm{G}$ concentration increases the wettability of the scaffold (D) AFM analysis revealed the average surface roughness of PCL and PCL-G0.05 nanocomposite showing an increase in the surface roughness of PCL with $G$ incorporation.

娄

Figure 2 
Material biocompatibility studies; (A-E) Cell viability of LIVE/DEAD cell staining, panel showed the merged pictures of coverslip, PCL only, PCL-G0.01, PCL-G0.05 and PCL- G0.1 (evaluation of all the materials was repeated for three times in triplicates).(F-J) SEM images for evaluation of MSCs characteristic morphology on PCL and PCL-G nanocomposite

原

\section{Figure 2}

Material biocompatibility studies; (A-E) Cell viability of LIVE/DEAD cell staining, panel showed the merged pictures of coverslip, PCL only, PCL-G0.01, PCL-G0.05 and PCL- G0.1 (evaluation of all the materials was repeated for three times in triplicates).(F-J) SEM images for evaluation of MSCs characteristic morphology on PCL and PCL-G nanocomposite
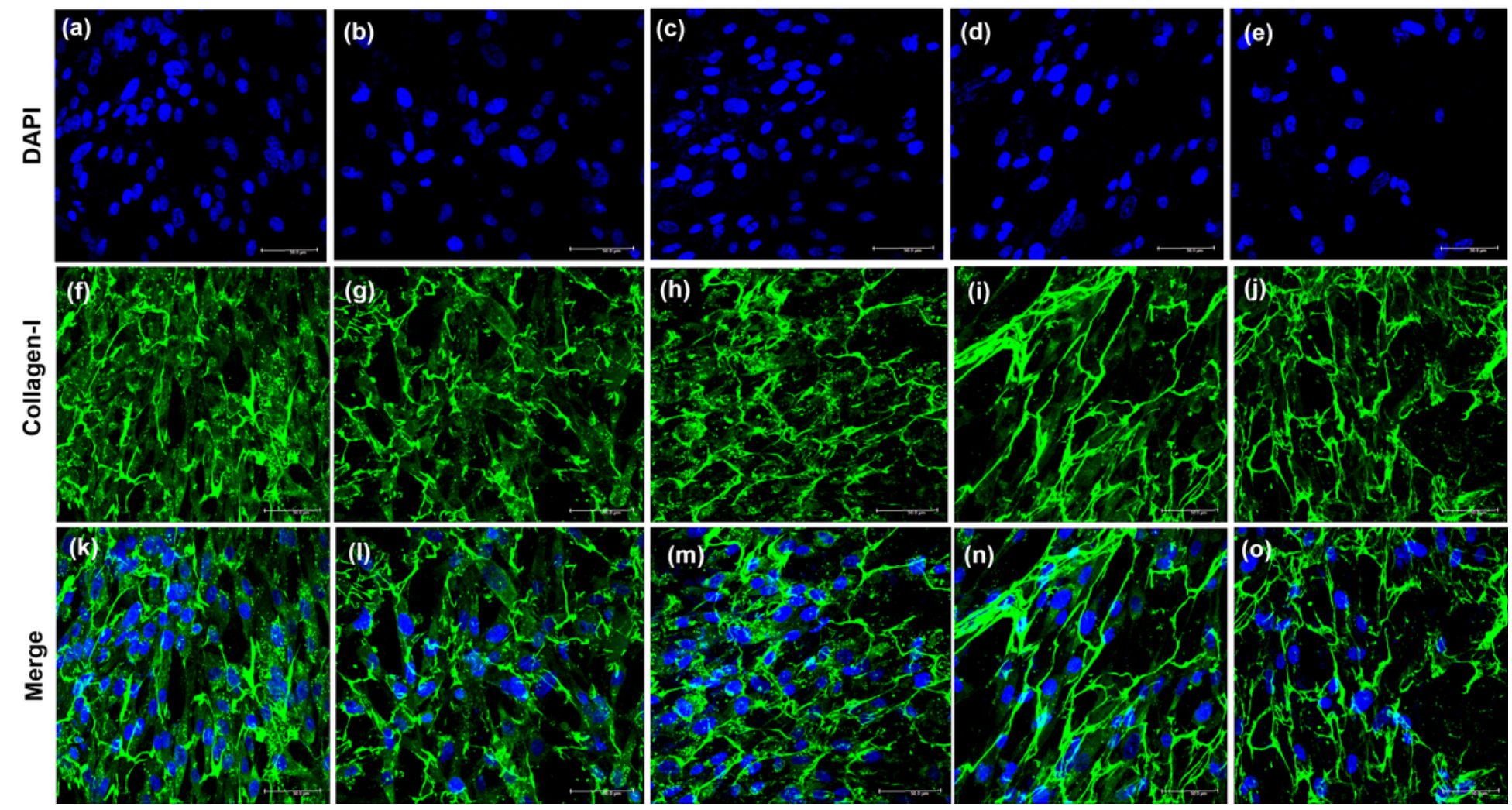

Figure 3

Representative images shows immunofluorescent staining for collagen-I on glass cover surface, $\mathrm{PCL}$, PCL-G0.01, PCL- G0.05 and PCL-G0.1 

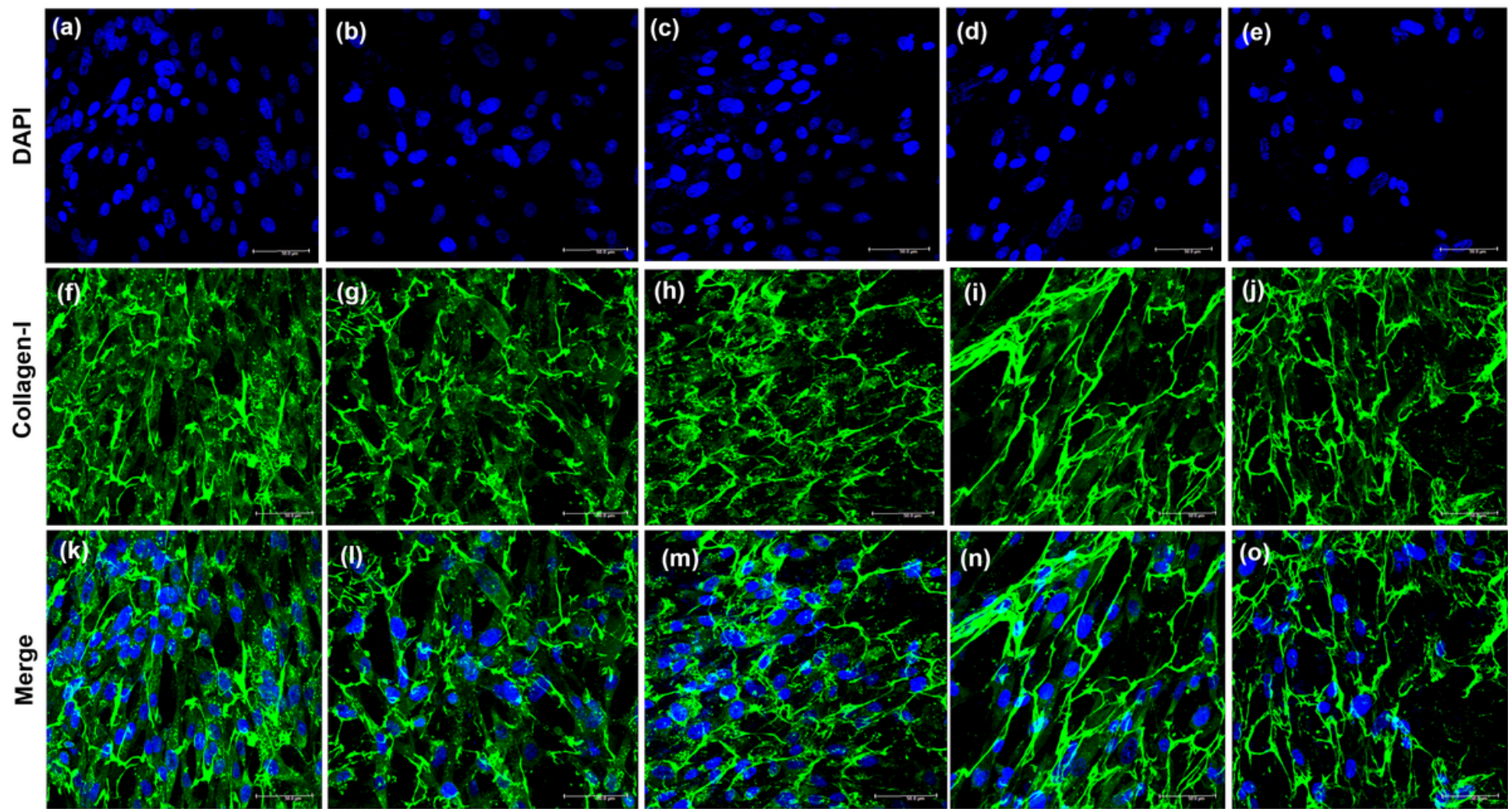

Figure 3

Representative images shows immunofluorescent staining for collagen-I on glass cover surface, $\mathrm{PCL}$, PCL-G0.01, PCL- G0.05 and PCL-G0.1 

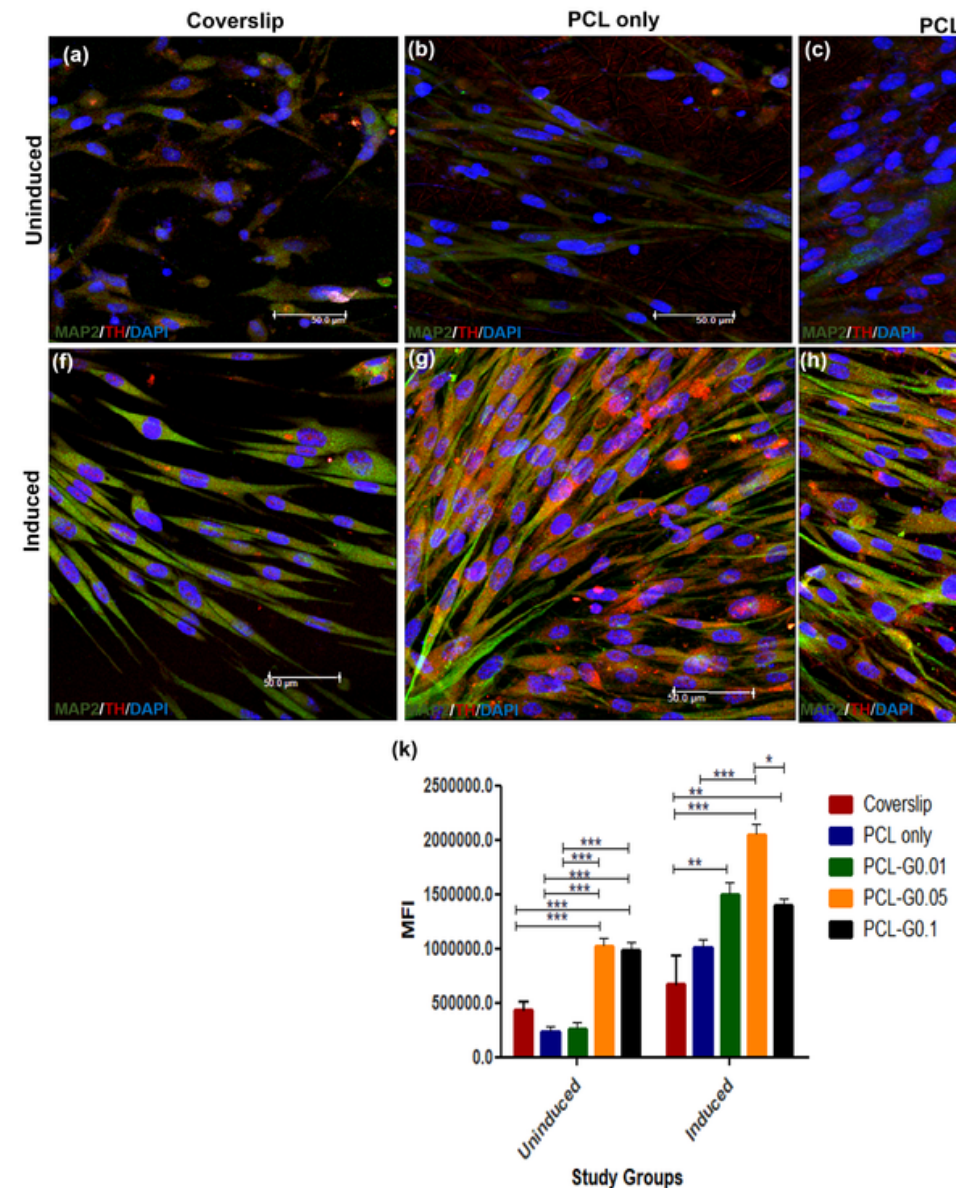

PCL-G0.01

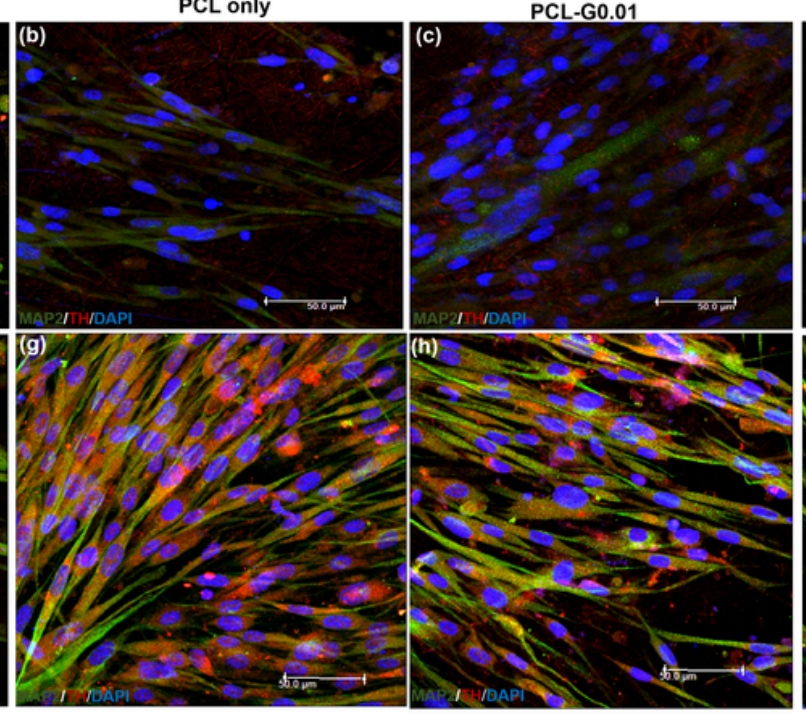

(I)
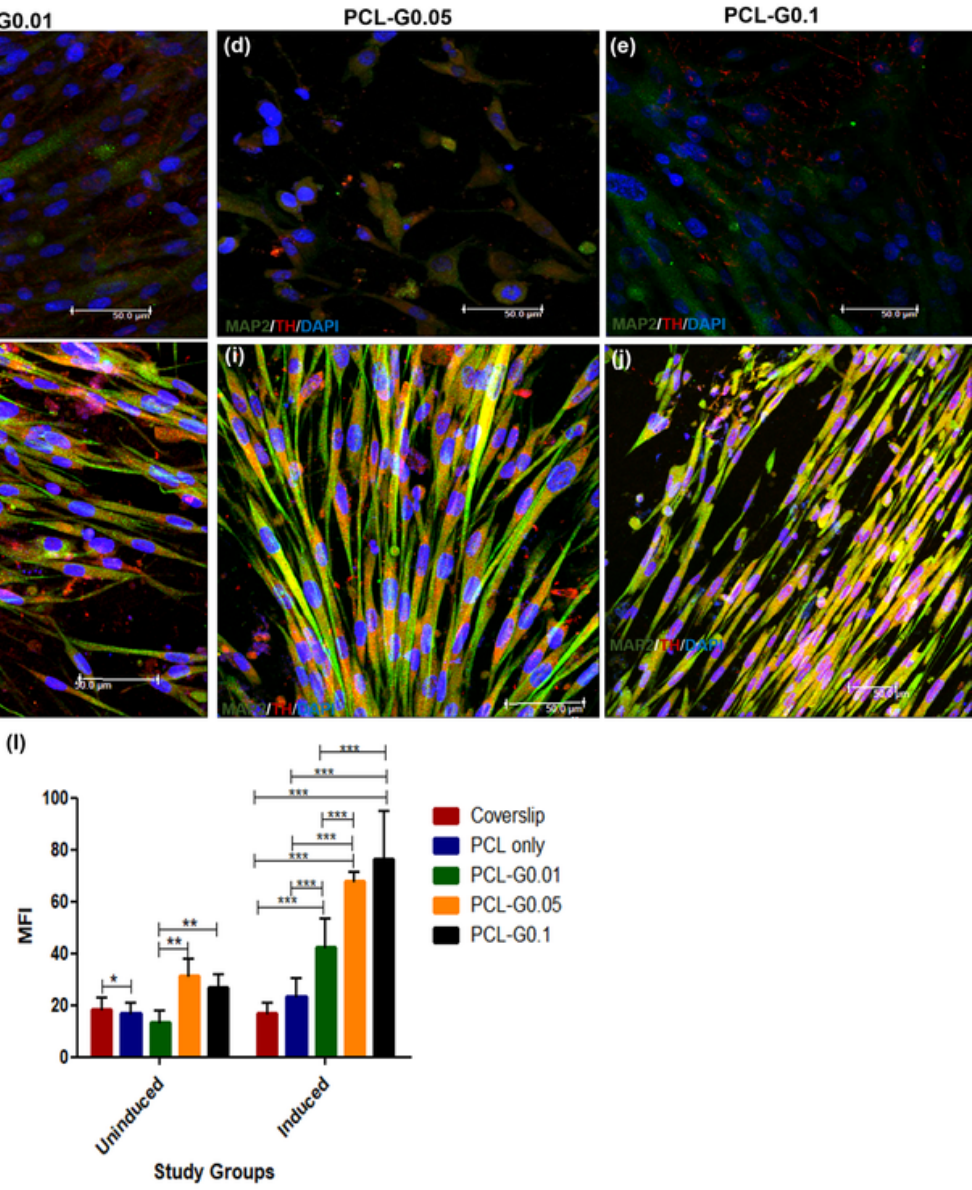

Figure 4

To study the relative impact of contact guidance on neurite length outgrowth, we investigated whether the electrospun mess organization affects the cells orientation or/and neuronal network. 

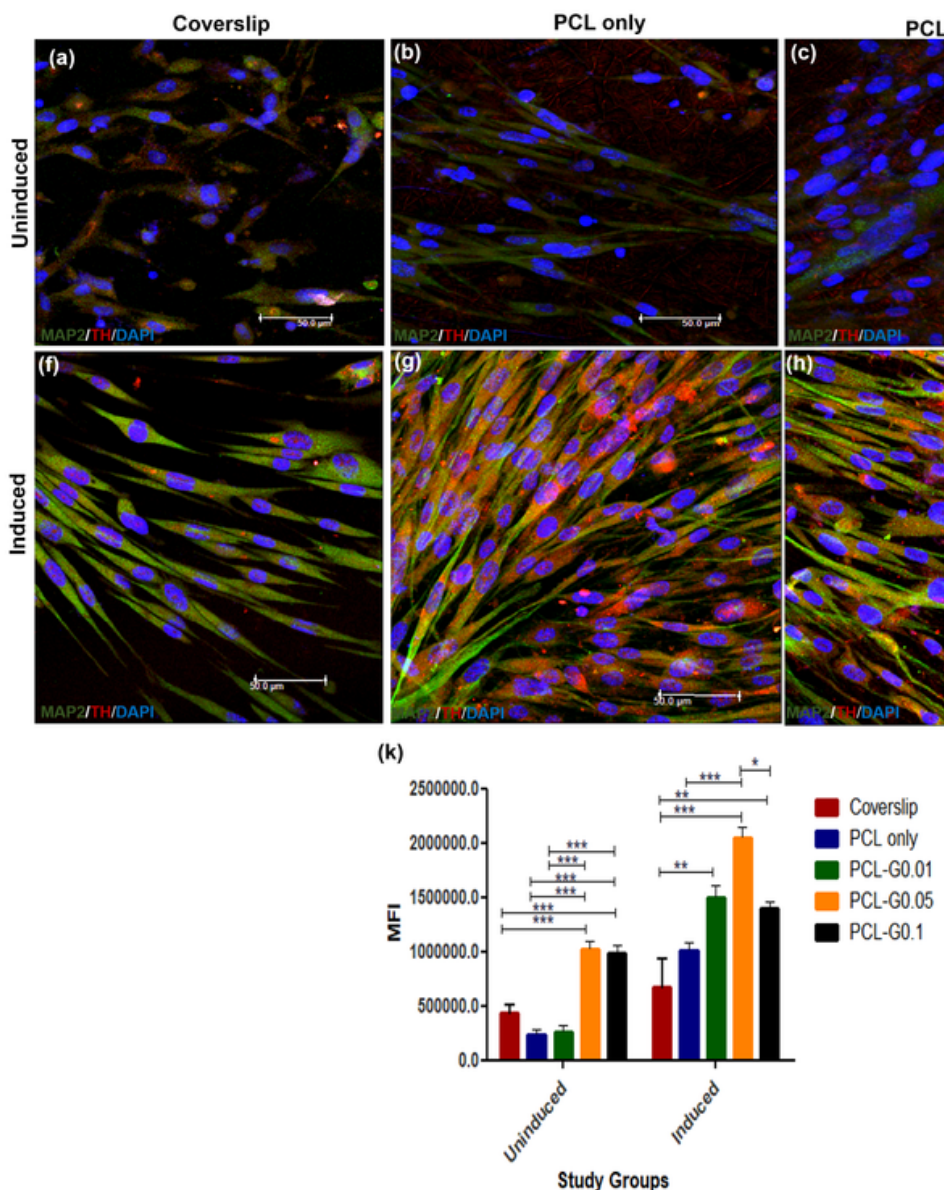

PCL only

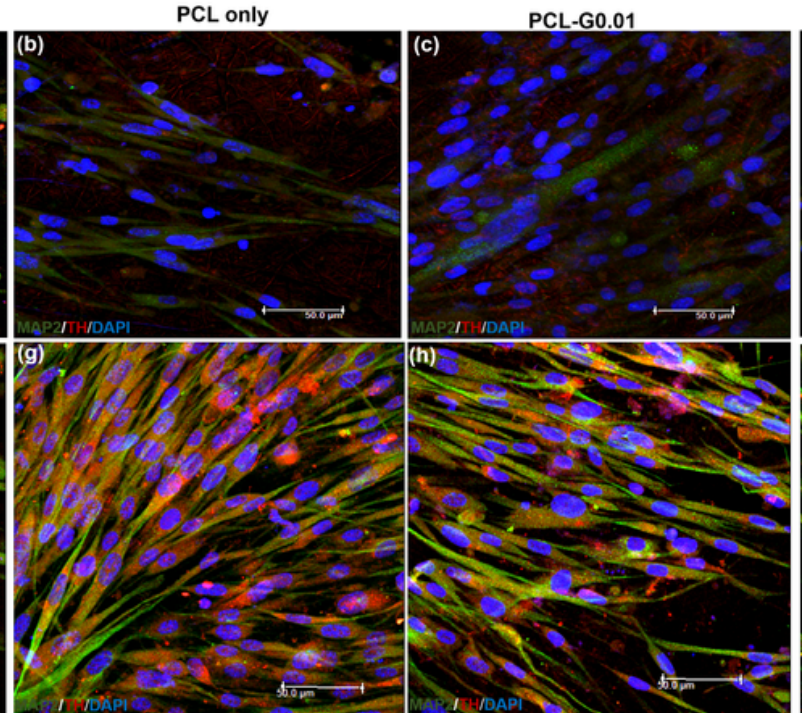

(I)

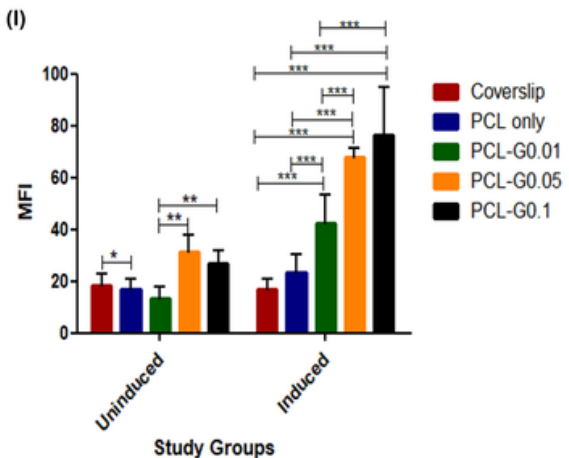

Figure 4

To study the relative impact of contact guidance on neurite length outgrowth, we investigated whether the electrospun mess organization affects the cells orientation or/and neuronal network. 

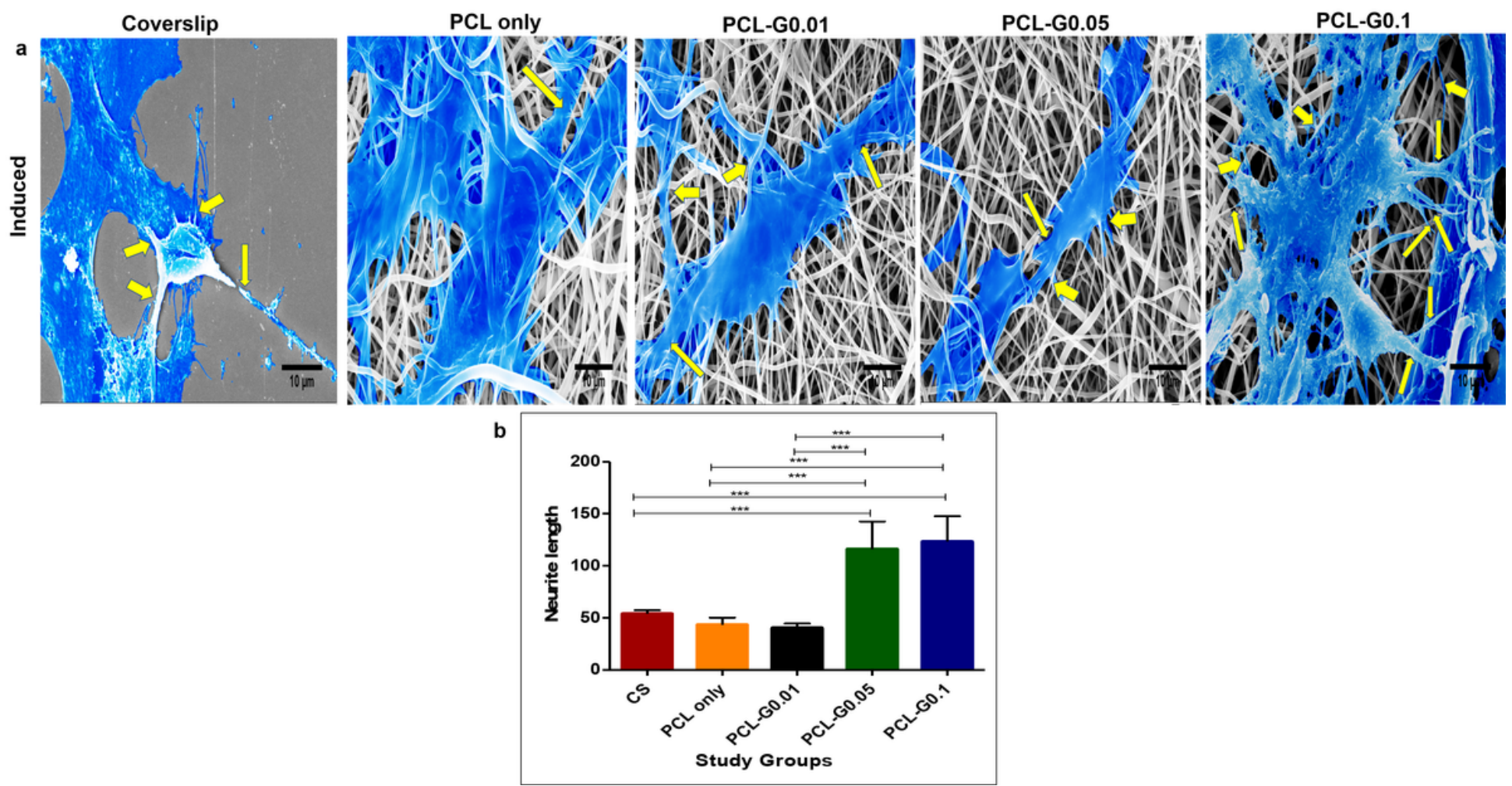

Figure 5

Characteristic morphology of neurons on PCL and PCL-G nanocomposite; (A) SEM micrographs depicting morphological changes in hMSCs upon induction to neuronal lineage on PCL and PCL-G. Scale bar 10 micron. (B), (C) Representative images for showing the neurite length variation in PCL and PCL-G scaffolds.
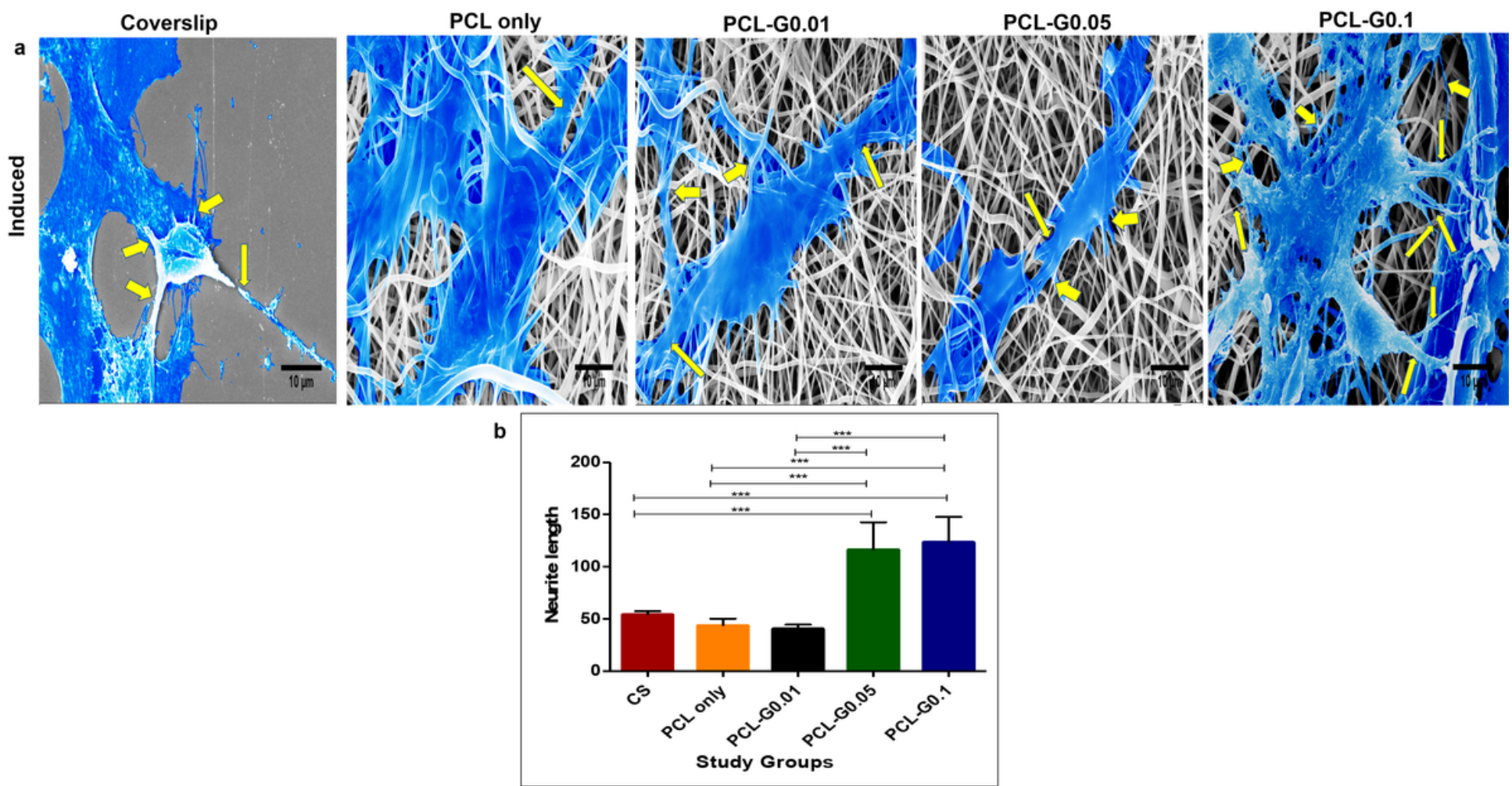


\section{Figure 5}

Characteristic morphology of neurons on PCL and PCL-G nanocomposite; (A) SEM micrographs depicting morphological changes in hMSCs upon induction to neuronal lineage on PCL and PCL-G. Scale bar 10 micron. (B), (C) Representative images for showing the neurite length variation in PCL and PCL-G scaffolds.
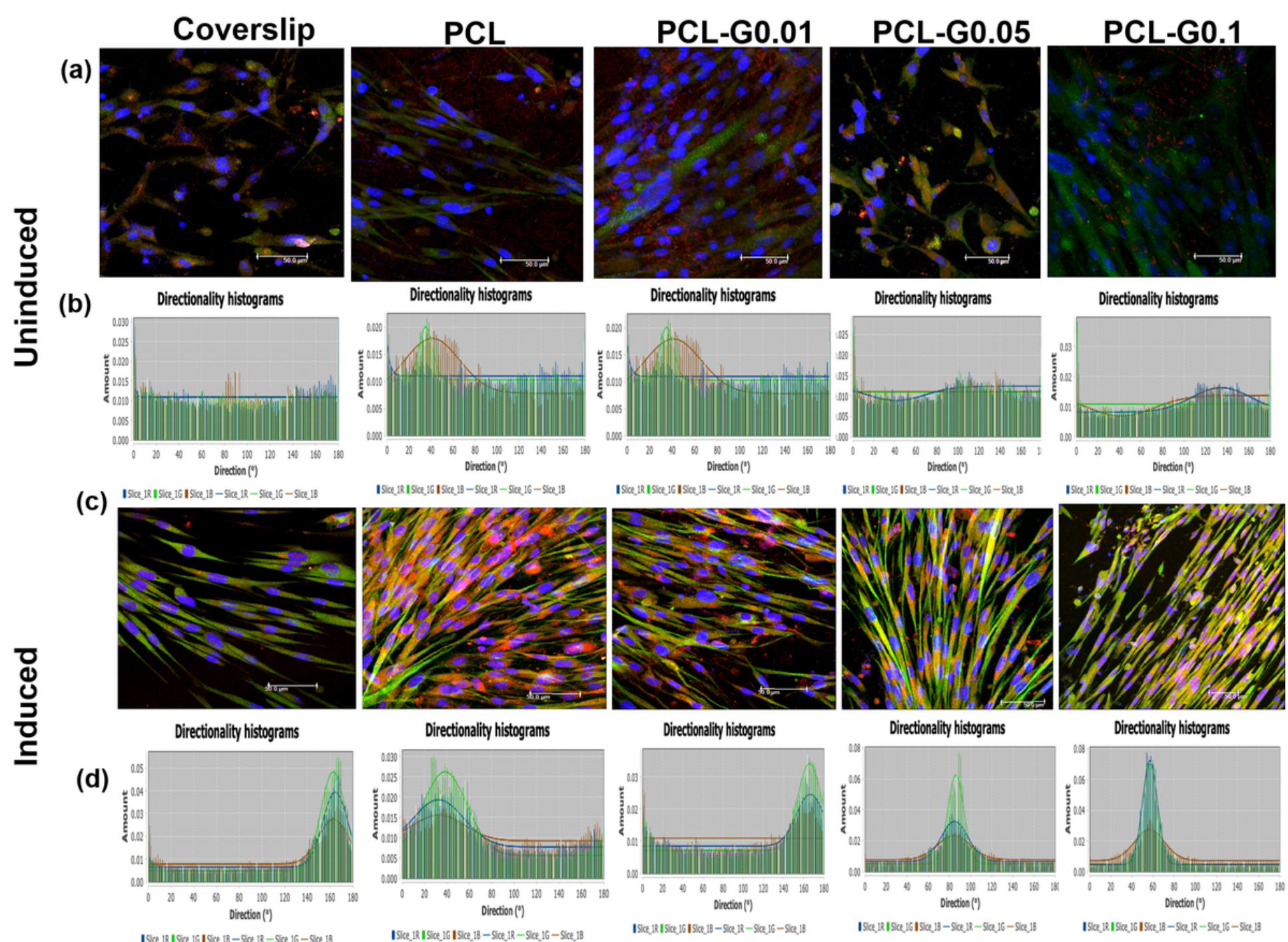

Directionality histograms

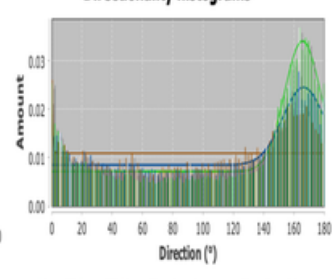

Directionality histograms

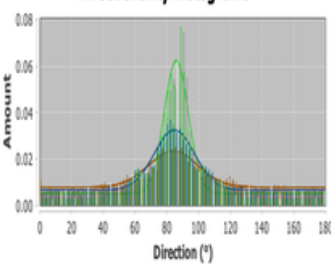

Directionality histograms

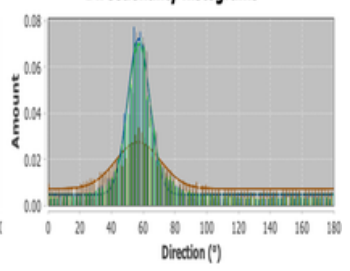

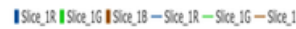

\section{Figure 6}

Representative images showing the increase in degree of cell orientation in (A-B) un-induced verses (C-D) Induced group over all the nanocomposite. All data are displayed as mean \pm standard deviation. 

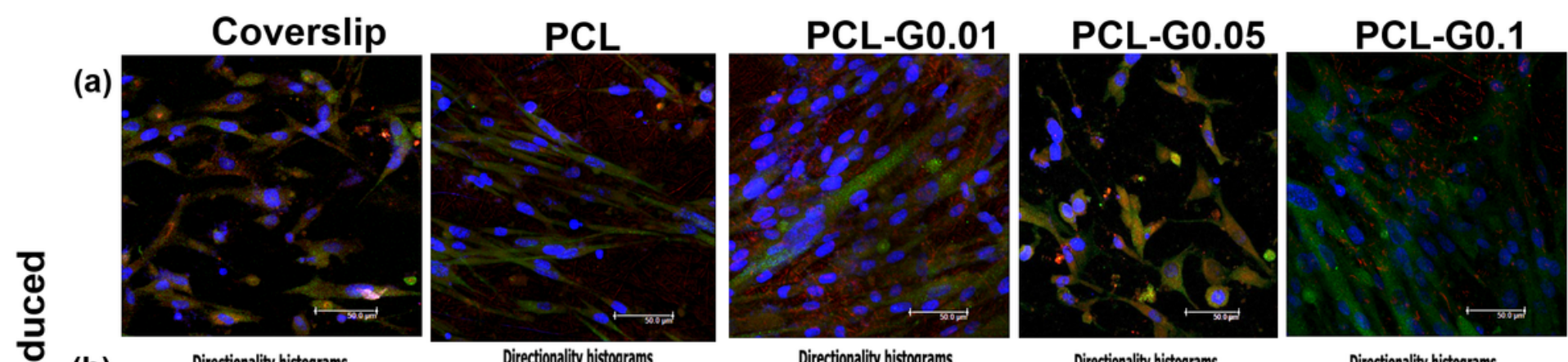

(b)
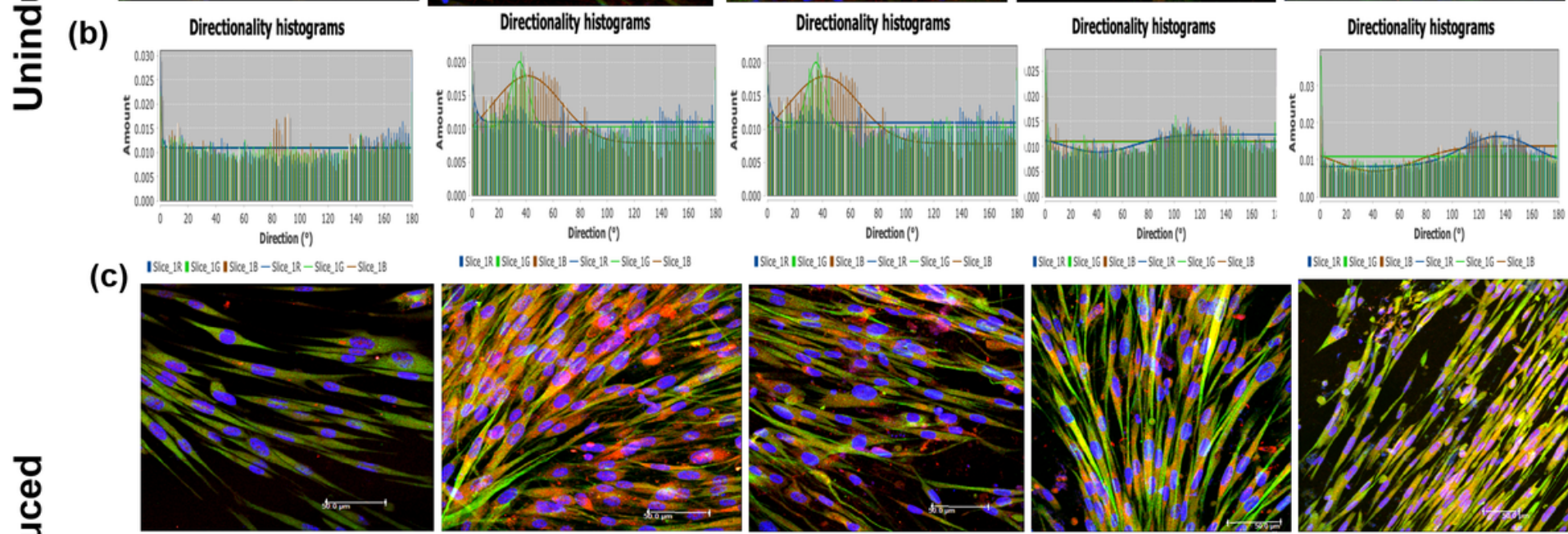

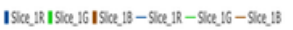

(d)
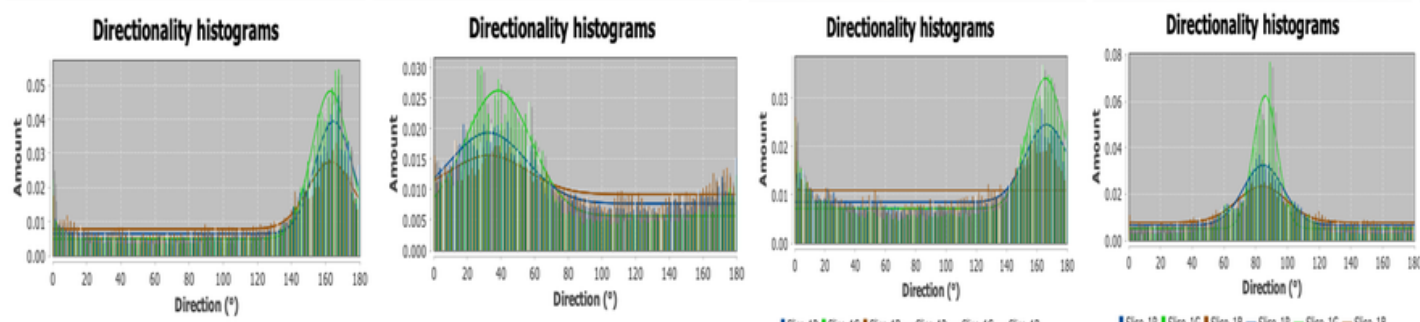

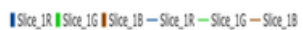

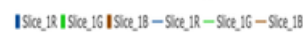
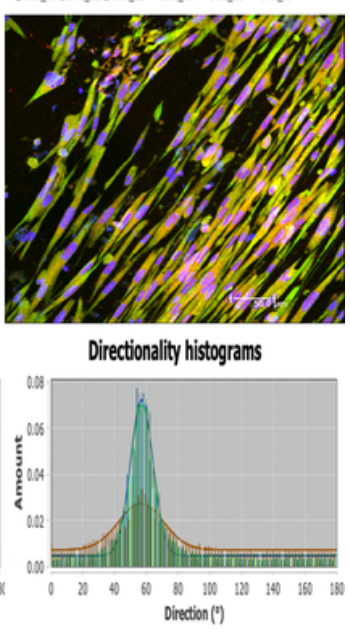

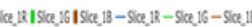

\section{Figure 6}

Representative images showing the increase in degree of cell orientation in (A-B) un-induced verses (C-D) Induced group over all the nanocomposite. All data are displayed as mean \pm standard deviation. 
(a)

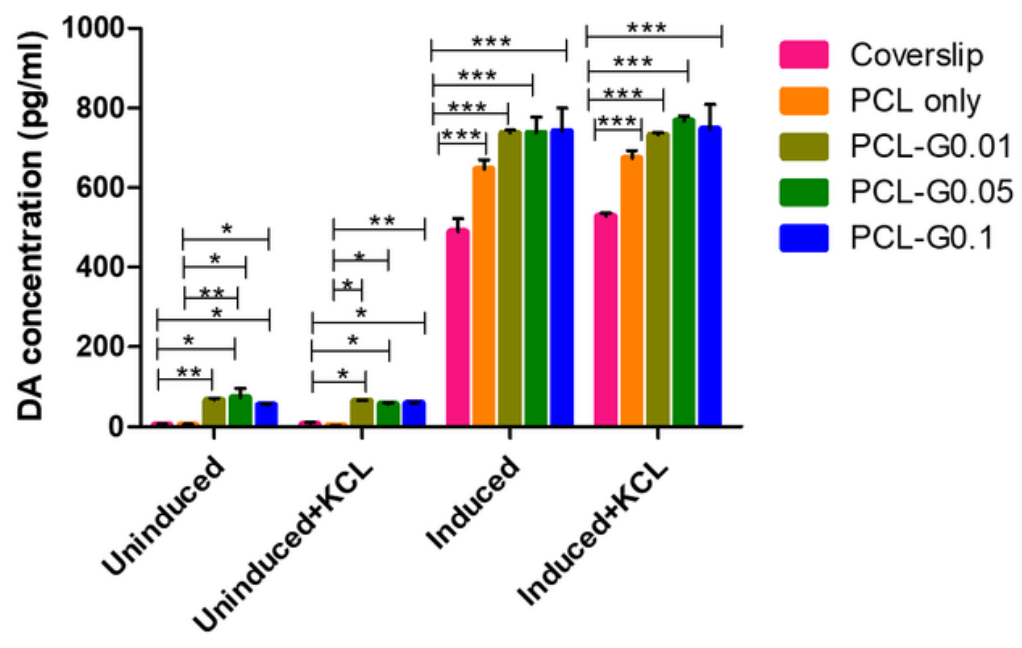

Study Groups

(b)

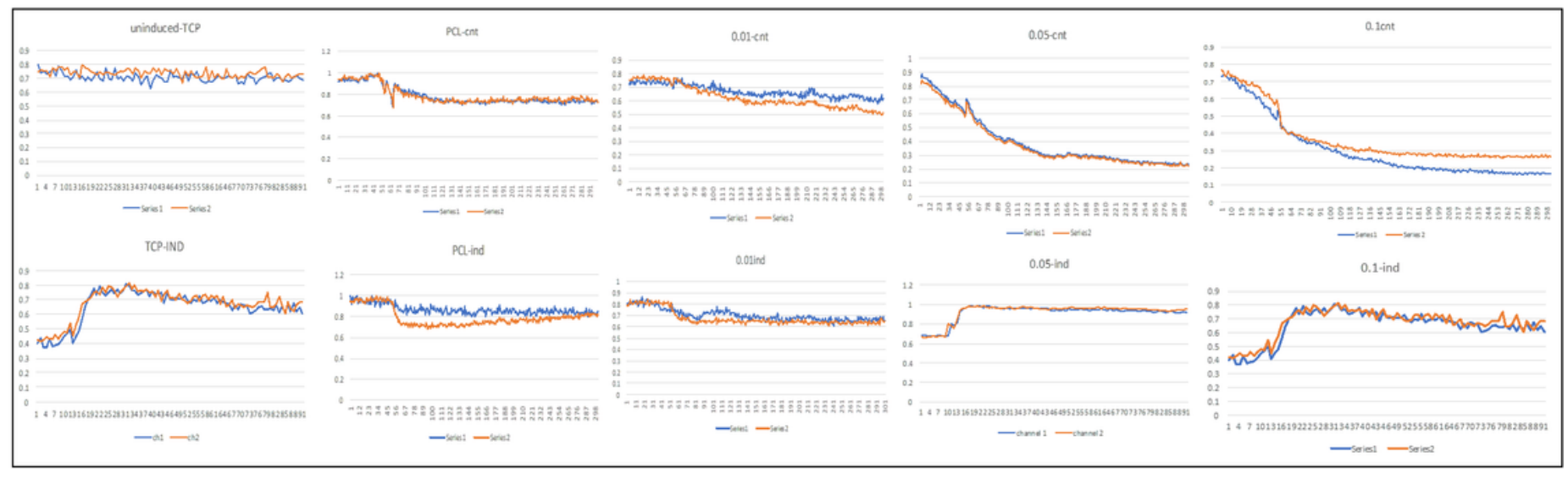

\section{Figure 7}

Functionality of hMSCs differentiated dopaminergic neurons. (A) A bar graph represents the release of dopamine by ELISA in different condition and study groups. (B) A line graph represents the time point at which calcium ion efflux changed after addition of KCL. All data are displayed as mean \pm standard deviation. ${ }^{*} \mathrm{p}<0.001$ compared with Coverslip/PCL/PCL-G0.01/PCL- G0.05/PCL-G0.1 (the statistical test is two-way). 


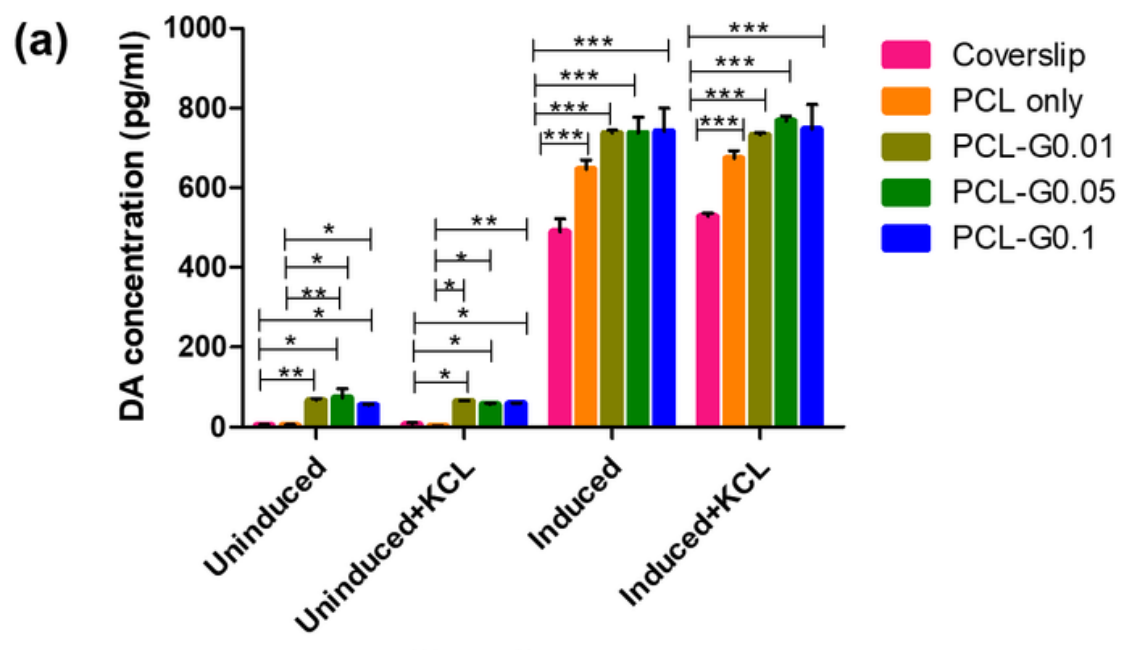

(b)

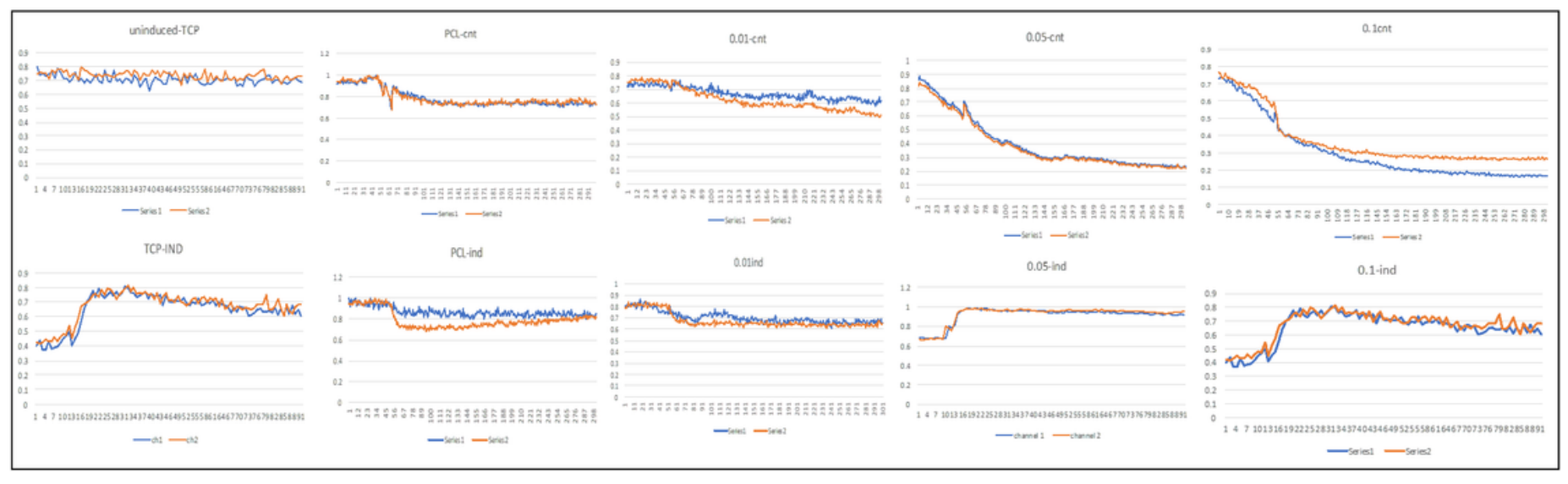

Figure 7

Functionality of hMSCs differentiated dopaminergic neurons. (A) A bar graph represents the release of dopamine by ELISA in different condition and study groups. (B) A line graph represents the time point at which calcium ion efflux changed after addition of KCL. All data are displayed as mean \pm standard deviation. ${ }^{*} p<0.001$ compared with Coverslip/PCL/PCL-G0.01/PCL- G0.05/PCL-G0.1 (the statistical test is two-way). 
(a)

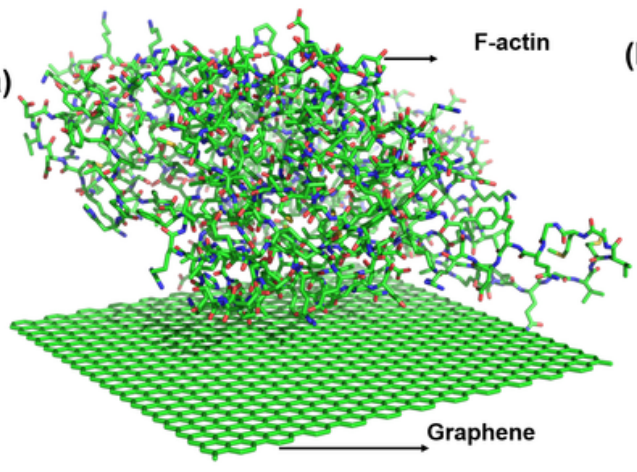

(d)

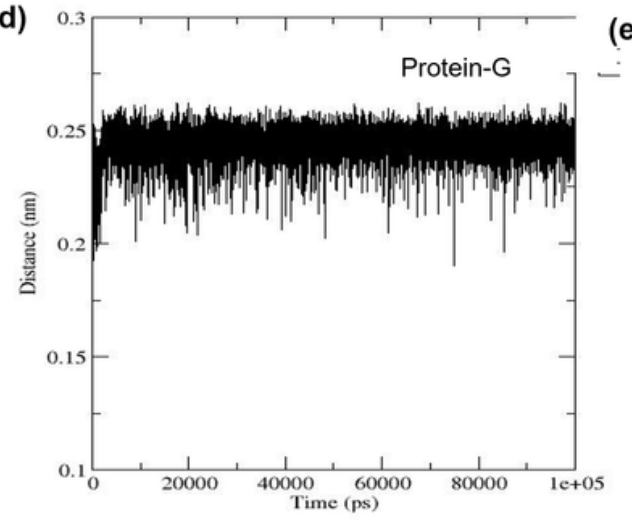

(b)

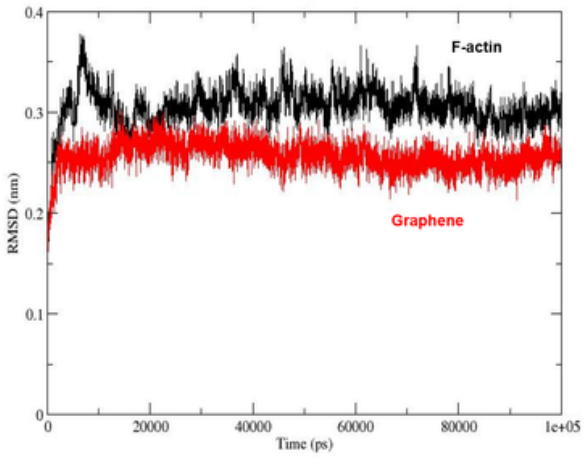

(e)

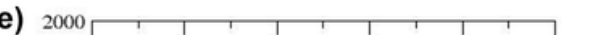

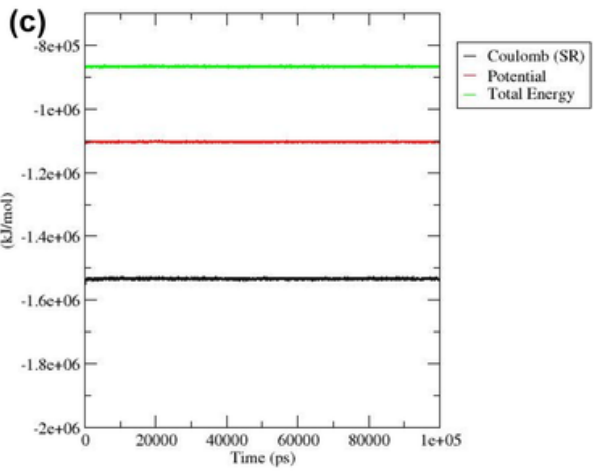

(f)

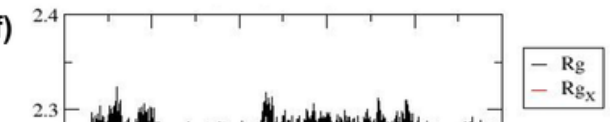

\section{Figure 8}

MD simulation studies for $\mathrm{G}$ and actin interaction; (A) The estimated free energy of F-actin binding with armchair GRA was calculated by intermolecular energy (1), total internal energy (2), torsional energy (3) and unbound energy (4) using equation $\{(1)+(2)+(3)-(4)\}$. (B) RMSD, (C) Gromacs energies, (D) minimum distance, $(E)$ number of contacts established within $0.4 \mathrm{~nm}$ between F-actin and GRA, and (F) compactness of F-actin $(\mathrm{Rg})$ and GRA (Rgx) analyzed by radius of gyration 
(a)

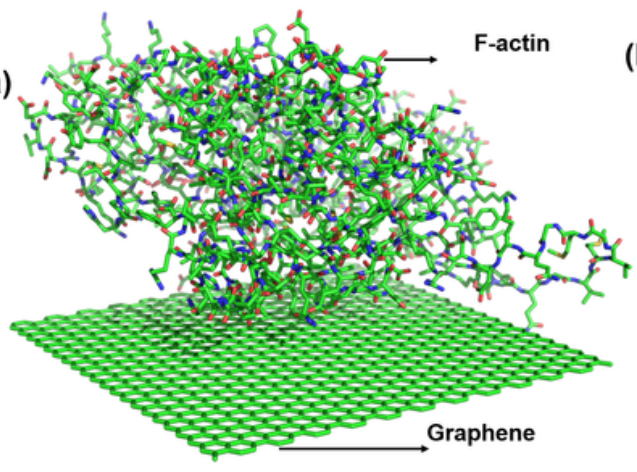

(d)

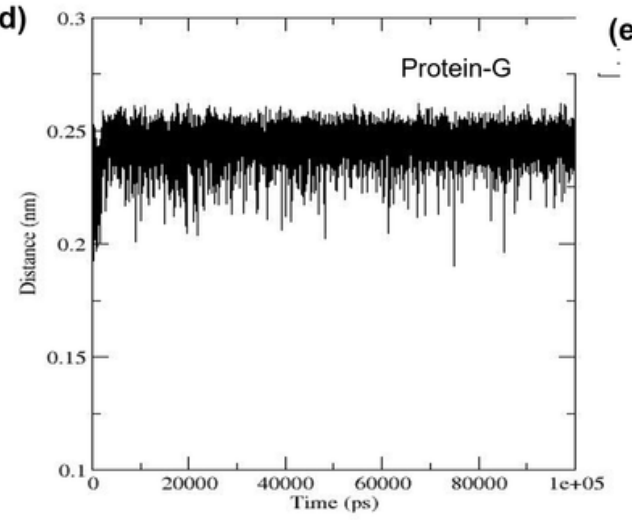

(b)

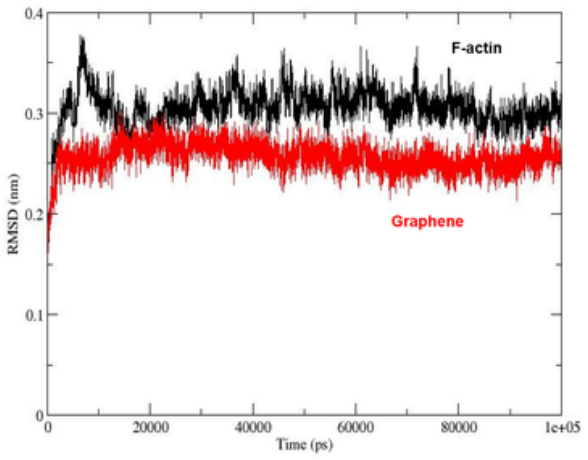

(e)

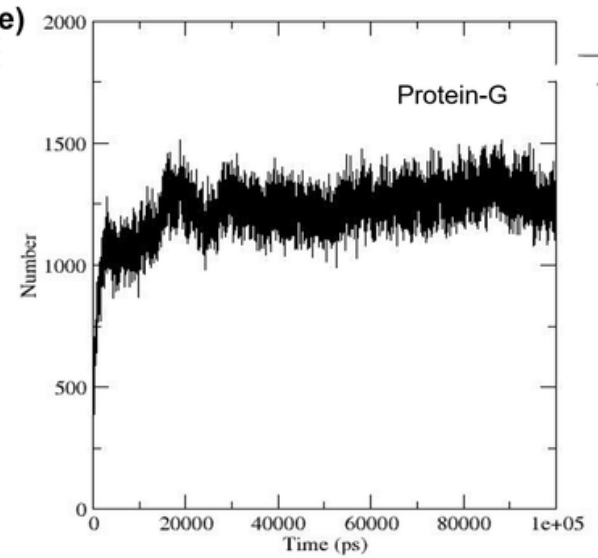

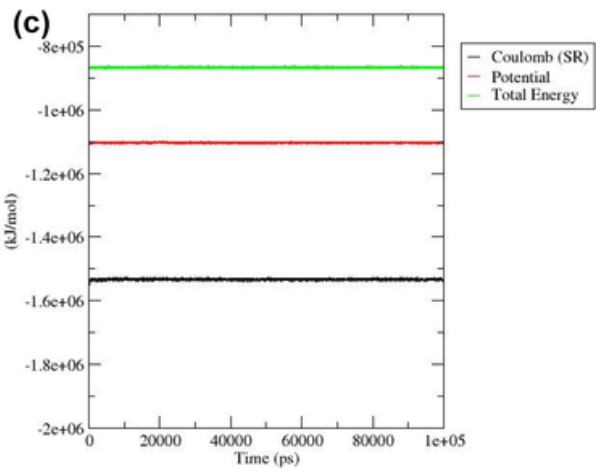

(f)

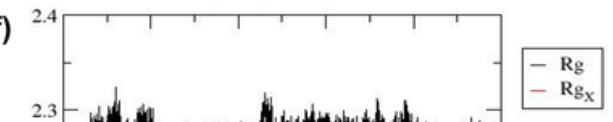

\section{Figure 8}

MD simulation studies for $\mathrm{G}$ and actin interaction; (A) The estimated free energy of F-actin binding with armchair GRA was calculated by intermolecular energy (1), total internal energy (2), torsional energy (3) and unbound energy (4) using equation $\{(1)+(2)+(3)-(4)\}$. (B) RMSD, (C) Gromacs energies, (D) minimum distance, $(E)$ number of contacts established within $0.4 \mathrm{~nm}$ between F-actin and GRA, and (F) compactness of F-actin ( $\mathrm{Rg})$ and GRA (Rgx) analyzed by radius of gyration

\section{Supplementary Files}

This is a list of supplementary files associated with this preprint. Click to download.

- Supplementarydraft.docx

- Supplementarydraft.docx 